TRANSACTIONS OF THE

AMERICAN MATHEMATICAL SOCIETY

Volume 363, Number 5, May 2011, Pages 2551-2569

S 0002-9947(2010)05134-7

Article electronically published on December 15, 2010

\title{
1-POINT GROMOV-WITTEN INVARIANTS OF THE MODULI SPACES OF SHEAVES OVER THE PROJECTIVE PLANE
}

\author{
WEI-PING LI AND ZHENBO QIN
}

\begin{abstract}
The Gieseker-Uhlenbeck morphism maps the Gieseker moduli space of stable rank-2 sheaves on a smooth projective surface to the Uhlenbeck compactification and is a generalization of the Hilbert-Chow morphism for Hilbert schemes of points. When the surface is the complex projective plane, we determine all the 1-point genus-0 Gromov-Witten invariants extremal with respect to the Gieseker-Uhlenbeck morphism. The main idea is to understand the virtual fundamental class of the moduli space of stable maps by studying the obstruction sheaf and using a meromorphic 2-form on the Gieseker moduli space.
\end{abstract}

\section{INTRODUCTION}

Recently there has been intensive interest in studying the quantum cohomology and Gromov-Witten theory of Hilbert schemes of points on algebraic surfaces. Two main reasons are the connections with the Donaldson-Thomas theory of 3-folds and with Ruan's Cohomological Crepant Resolution Conjecture. Roughly speaking, the Crepant Resolution Conjecture asserts that the quantum cohomology of an orbifold $Z$ coincides with the quantum cohomology of a crepant resolution $Y$ of $Z$ after analytic continuation and specialization of quantum parameters. For an algebraic surface $X$, let $X^{[n]}$ be the Hilbert scheme of $n$ points on $X$ and $\operatorname{Sym}^{n}(X)$ be the $n$-th symmetric product of $X$. It is well known that $X^{[n]}$ is smooth of dimension $2 n$ and the Hilbert-Chow morphism $\Phi: X^{[n]} \rightarrow \operatorname{Sym}^{n}(X)$ is a crepant resolution of the global orbifold $\operatorname{Sym}^{n}(X)$.

A natural generalization of the Hilbert-Chow morphism $\Phi$ is the Gieseker-Uhlenbeck morphism $\Psi$ from the moduli space of Gieseker semistable rank-2 torsion-free sheaves on $X$ to the Uhlenbeck compactification space. This morphism was constructed in [LJ1, Mor, and was shown to be crepant [LJ2, Q-Z] when the Gieseker moduli space is smooth. For the projective plane $X=\mathbb{P}^{2}$, the moduli space $\overline{\mathfrak{M}}(n)$ of Gieseker semistable sheaves $V$ on $X$ with $c_{1}(V)=-1$ and $c_{2}(V)=n$ is a smooth irreducible projective variety of dimension $4 n-4$ when $n \geq 1$. In Q-Z, it is proved that there is exactly one primitive integral class $\mathfrak{f} \in H_{2}(\overline{\mathfrak{M}}(n) ; \mathbb{Z})$ contracted by the Gieseker-Uhlenbeck morphism $\Psi: \overline{\mathfrak{M}}(n) \rightarrow \overline{\mathfrak{U}}(n)$.

Received by the editors February 6, 2009 and, in revised form, June 1, 2009.

2000 Mathematics Subject Classification. Primary 14D20, 14 N35.

Key words and phrases. Gieseker moduli spaces, Gromov-Witten invariants.

The first author was partially supported by the grants GRF601905 and GRF601808.

The second author was partially supported by an NSF grant.

(C)2010 American Mathematical Society 
The goal of this paper is to determine all the 1-point genus-0 Gromov-Witten invariants $\langle\alpha\rangle_{0, d \mathfrak{f}}, \alpha \in H^{8 n-12}(\overline{\mathfrak{M}}(n) ; \mathbb{C})$ extremal with respect to $\Psi$ for $n \geq 3$. When $n \geq 3$, the homology group $H_{4}(\overline{\mathfrak{M}}(n) ; \mathbb{C})$ is of rank 6 , and a basis is given by $\left\{\Xi_{1}, \ldots, \Xi_{6}\right\}$ (see $\varangle$ for details). The Poincaré duals $\operatorname{PD}\left(\Xi_{1}\right), \ldots, \operatorname{PD}\left(\Xi_{6}\right)$ form a basis of $H^{8 n-12}(\overline{\mathfrak{M}}(n) ; \mathbb{C})$.

Theorem 1.1. Let $d \geq 1$ and $n \geq 3$. The Gromov-Witten invariants $\langle\alpha\rangle_{0, d f}$ for the classes $\alpha=\operatorname{PD}\left(\Xi_{1}\right), \ldots, \operatorname{PD}\left(\Xi_{6}\right) \in H^{8 n-12}(\overline{\mathfrak{M}}(n) ; \mathbb{C})$ are respectively equal to

$$
-6 / d^{2}, \quad 12 / d^{2}, \quad 0, \quad-6 / d^{2}, \quad 0, \quad 0 .
$$

When $n=1$, the moduli space $\overline{\mathfrak{M}}(n)$ is a point. When $n=2$, the fourth Betti number $b_{4}$ of the moduli space $\overline{\mathfrak{M}}(n)$ is equal to 3 , which is different from the case $n \geq 3$. The result for $n=2$ will appear elsewhere via a different method (see Remark 3.2.

An interesting observation is that the 1-point genus-0 Gromov-Witten invariants $\langle\alpha\rangle_{0, d f}$ are independent of the second Chern class $n$.

Conjecture 1.2. Let $d \geq 1$ and $n \geq 3$. Then the extremal genus-0 Gromov-Witten invariants $\left\langle\alpha_{1}, \ldots, \alpha_{k}\right\rangle_{0, d \mathfrak{f}}$ of the moduli space $\overline{\mathfrak{M}}(n)$ are independent of $n$.

There are two main ideas in our proof of Theorem 1.1. The first one is to determine the restriction of the obstruction sheaf of the Gromov-Witten theory for $\overline{\mathfrak{M}}(n)$ to certain open subsets of the moduli space $\overline{\mathfrak{M}}_{0,1}(\overline{\mathfrak{M}}(n), d \mathfrak{f})$ of stable maps. This enables us to determine the 1-point invariants $\langle\alpha\rangle_{0, d \mathfrak{f}}$ for the first four cohomology classes $\alpha=\operatorname{PD}\left(\Xi_{1}\right), \ldots, \operatorname{PD}\left(\Xi_{4}\right)$.

The second one is to study the support of the virtual fundamental class

$$
\left[\overline{\mathfrak{M}}_{0,1}(\overline{\mathfrak{M}}(n), d \mathfrak{f})\right]^{\text {vir }} \in A_{4 n-6}\left(\overline{\mathfrak{M}}_{0,1}(\overline{\mathfrak{M}}(n), d \mathfrak{f})\right)
$$

using the techniques developed in $[\mathrm{K}-\mathrm{L}, \mathrm{L}-\mathrm{L}$. By introducing a suitable meromorphic 2-form $\Theta$ on the Gieseker moduli space $\overline{\mathfrak{M}}(n)$, we show that

$$
e v_{1}\left(\operatorname{Supp}\left(\left[\overline{\mathfrak{M}}_{0,1}(\overline{\mathfrak{M}}(n), d \mathfrak{f})\right]^{\mathrm{vir}}\right)\right) \subset \mathfrak{T}_{C_{0}}(n) \coprod \mathfrak{U}_{C_{0}}(n),
$$

where $e v_{1}: \overline{\mathfrak{M}}_{0,1}(\overline{\mathfrak{M}}(n), d \mathfrak{f}) \rightarrow \overline{\mathfrak{M}}(n)$ is the evaluation map, and $\mathfrak{T}_{C_{0}}(n)$ (respectively, $\left.\mathfrak{U}_{C_{0}}(n)\right)$ is the subset of $\overline{\mathfrak{M}}(n)$ consisting of all the nonlocally free sheaves $V$ such that $\left.V\right|_{C_{0}}$ contains torsion (respectively, $\left.V\right|_{C_{0}}$ is torsion-free and unstable). This allows us to show that $\langle\alpha\rangle_{0, d f}=0$ for $\alpha=\operatorname{PD}\left(\Xi_{5}\right), \operatorname{PD}\left(\Xi_{6}\right)$.

This paper is organized as follows. In $₫ 2$, the Gromov-Witten theory is reviewed. In $₫ 3$, we recall some properties of the Gieseker moduli space $\overline{\mathfrak{M}}(n)$ and the GiesekerUhlenbeck morphism $\Psi$. We study the boundary divisor of $\overline{\mathfrak{M}}(n)$ consisting of nonlocally free sheaves in $\overline{\mathfrak{M}}(n)$. In 44 , the basis $\left\{\Xi_{1}, \ldots, \Xi_{6}\right\}$ for $H_{4}(\overline{\mathfrak{M}}(n) ; \mathbb{C})$ is constructed. In 95 , we analyze the obstruction sheaf of the Gromov-Witten theory for $\overline{\mathfrak{M}}(n)$. In 96 , (1.1) is proved. In $₫ 7$, we verify Theorem 1.1 .

\section{Stable maps and Gromov-Witten invariants}

Let $Y$ be a smooth projective variety. A $k$-pointed stable map to $Y$ consists of a complete nodal curve $D$ with $k$ distinct ordered smooth points $p_{1}, \ldots, p_{k}$ and a morphism $\mu: D \rightarrow Y$ such that the data $\left(\mu, D, p_{1}, \ldots, p_{k}\right)$ has only finitely many automorphisms. In this case, the stable map is denoted by $\left[\mu:\left(D ; p_{1}, \ldots, p_{k}\right) \rightarrow Y\right]$. For a fixed homology class $\beta \in H_{2}(Y, \mathbb{Z})$, let $\overline{\mathfrak{M}}_{g, k}(Y, \beta)$ be the coarse moduli space 
parameterizing all the stable maps $\left[\mu:\left(D ; p_{1}, \ldots, p_{k}\right) \rightarrow Y\right]$ such that $\mu_{*}[D]=\beta$ and the arithmetic genus of $D$ is $g$. Then, we have the evaluation map:

$$
e v_{k}: \overline{\mathfrak{M}}_{g, k}(Y, \beta) \rightarrow Y^{k}
$$

defined by $e v_{k}\left(\left[\mu:\left(D ; p_{1}, \ldots, p_{k}\right) \rightarrow Y\right]\right)=\left(\mu\left(p_{1}\right), \ldots, \mu\left(p_{k}\right)\right)$. It is known [F-P, LT1, LT2, Beh, B-F that the coarse moduli space $\overline{\mathfrak{M}}_{g, k}(Y, \beta)$ is projective and has a virtual fundamental class $\left[\overline{\mathfrak{M}}_{g, k}(Y, \beta)\right]^{\text {vir }} \in A_{\mathfrak{d}}\left(\overline{\mathfrak{M}}_{g, k}(Y, \beta)\right)$, where

$$
\mathfrak{d}=-\left(K_{Y} \cdot \beta\right)+(\operatorname{dim}(Y)-3)(1-g)+k
$$

is the expected complex dimension of $\overline{\mathfrak{M}}_{g, k}(Y, \beta)$, and $A_{\mathfrak{d}}\left(\overline{\mathfrak{M}}_{g, k}(Y, \beta)\right)$ is the Chow group of $\mathfrak{d}$-dimensional cycles in the moduli space $\overline{\mathfrak{M}}_{g, k}(Y, \beta)$.

The Gromov-Witten invariants are defined by using the virtual fundamental class $\left[\overline{\mathfrak{M}}_{g, k}(Y, \beta)\right]^{\text {vir }}$. Recall that an element $\alpha \in H^{*}(Y, \mathbb{C}) \stackrel{\text { def }}{=} \bigoplus_{j=0}^{2 \operatorname{dim}_{\mathbb{C}}(Y)} H^{j}(Y, \mathbb{C})$ is homogeneous if $\alpha \in H^{j}(Y, \mathbb{C})$ for some $j$; in this case, we take $|\alpha|=j$. Let $\alpha_{1}, \ldots, \alpha_{k} \in H^{*}(Y, \mathbb{C})$ such that every $\alpha_{i}$ is homogeneous and $\sum_{i=1}^{k}\left|\alpha_{i}\right|=2 \mathfrak{d}$. Then, we have the $k$-point Gromov-Witten invariant defined by

$$
\left\langle\alpha_{1}, \ldots, \alpha_{k}\right\rangle_{g, \beta}=\int_{\left[\overline{\mathfrak{M}}_{g, k}(Y, \beta)\right]^{\mathrm{vir}}} e v_{k}^{*}\left(\alpha_{1} \otimes \ldots \otimes \alpha_{k}\right) .
$$

Next, we summarize certain properties concerning the virtual fundamental class. To begin with, we recall that the excess dimension is the difference between the dimension of $\overline{\mathfrak{M}}_{g, k}(Y, \beta)$ and the expected dimension $\mathfrak{d}$ in (2.2). For $0 \leq i<k$, use

$$
f_{k, i}: \overline{\mathfrak{M}}_{g, k}(Y, \beta) \rightarrow \overline{\mathfrak{M}}_{g, i}(Y, \beta)
$$

to stand for the forgetful map obtained by forgetting the last $k-i$ marked points and contracting all the unstable components. It is known that $f_{k, i}$ is flat when $\beta \neq 0$ and $0 \leq i<k$. The following can be found in LT1, Beh, Get, $\mathrm{C}-\mathrm{K}$.

Proposition 2.1. Let $\beta \in H_{2}(Y, \mathbb{Z})$ and $\beta \neq 0$. Let $e$ be the excess dimension of $\overline{\mathfrak{M}}_{g, k}(Y, \beta)$, and $\mathfrak{M} \subset \overline{\mathfrak{M}}_{g, k}(Y, \beta)$ be a closed subscheme. Then,

(i) $\left[\overline{\mathfrak{M}}_{g, k}(Y, \beta)\right]^{\mathrm{vir}}=\left(f_{k, 0}\right)^{*}\left[\overline{\mathfrak{M}}_{g, 0}(Y, \beta)\right]^{\mathrm{vir}}$;

(ii) $\left[\overline{\mathfrak{M}}_{g, k}(Y, \beta)\right]^{\mathrm{vir}}=c_{e}\left(R^{1}\left(f_{k+1, k}\right)_{*}\left(e v_{k+1}\right)^{*} T_{Y}\right)$ if $R^{1}\left(f_{k+1, k}\right)_{*}\left(e v_{k+1}\right)^{*} T_{Y}$ is a rank-e locally free sheaf over the moduli space $\overline{\mathfrak{M}}_{g, k}(Y, \beta)$;

(iii) $\left.\left[\overline{\mathfrak{M}}_{g, k}(Y, \beta)\right]^{\mathrm{vir}}\right|_{\mathfrak{M}}=c_{e}\left(\left.\left(R^{1}\left(f_{k+1, k}\right)_{*}\left(e v_{k+1}\right)^{*} T_{Y}\right)\right|_{\mathfrak{M}}\right)$ if there exists an open subset $\mathfrak{O}$ of $\overline{\mathfrak{M}}_{g, k}(Y, \beta)$ such that $\mathfrak{M} \subset \mathfrak{O}$ (i.e, $\mathfrak{O}$ is an open neighborhood of $\mathfrak{M}$ ) and the restriction $\left.\left(R^{1}\left(f_{k+1, k}\right)_{*}\left(e v_{k+1}\right)^{*} T_{Y}\right)\right|_{\mathfrak{O}}$ is a rank-e locally free sheaf over $\mathfrak{O}$.

\section{The moduli SPace of Stable RanK-2 Sheaves on $\mathbb{P}^{2}$}

3.1. Some basic facts of the moduli space. Throughout the rest of this paper, let $X=\mathbb{P}^{2}$ be the projective plane, and let $\ell$ be a line in $X$. For an integer $n$, let $\overline{\mathfrak{M}}(n)$ be the moduli space parametrizing all Gieseker-semistable rank-2 sheaves $V$ over $X$ with $c_{1}(V)=-\ell, c_{2}(V)=n$. Note that every such sheaf $V$ is actually slope-stable and hence is Gieseker-stable. It is well known that, when $n \geq 1, \overline{\mathfrak{M}}(n)$ is nonempty, smooth, irreducible and rational with the expected dimension $4 n-4$; in addition, a universal sheaf over $\overline{\mathfrak{M}}(n) \times X$ exists. By Theorem 1 in $[\mathrm{Mar}$, the cohomology groups $H^{i}(\overline{\mathfrak{M}}(n) ; \mathbb{Z})$ are torsion-free for all $i$ and vanish for odd $i$. The 
same is true of the homology groups $H_{i}(\overline{\mathfrak{M}}(n) ; \mathbb{Z})$. Let $b_{i}(\overline{\mathfrak{M}}(n))$ be the $i$-th Betti number of $\overline{\mathfrak{M}}(n)$, and put

$$
p(\overline{\mathfrak{M}}(n) ; q)=\sum_{i=0}^{8 n-8} b_{i}(\overline{\mathfrak{M}}(n)) q^{i} .
$$

By Theorem 0.1 of $\overline{\mathrm{YoS}}, p(\overline{\mathfrak{M}}(n) ; q)$ equals the coefficient of $t^{n}$ of the series

$$
\begin{aligned}
& \frac{1}{\left(q^{2}-1\right) \cdot \sum_{n \in \mathbb{Z}} q^{2 n(2 n-1)} t^{n^{2}}} \cdot \sum_{b \geq 0}\left(\frac{q^{2(b+1)(2 b+1)}}{1-q^{8(b+1)} t^{2 b+1}}-\frac{q^{2 b(2 b+5)}}{1-q^{8 b} t^{2 b+1}}\right) t^{(b+1)^{2}} \\
& \cdot \prod_{d \geq 1} \frac{1}{\left(1-q^{4 d-2} t^{d}\right)^{2}\left(1-q^{4 d} t^{d}\right)^{2}\left(1-q^{4 d+2} t^{d}\right)^{2}} .
\end{aligned}
$$

In the rest of the subsection, we review Strømme's work in $[\mathrm{Str}$ and give a basis of $H^{4}(\overline{\mathfrak{M}}(n), \mathbb{Z})$ in terms of the classes from $\left[\mathrm{Str}\right.$. A basis of $H_{4}(\overline{\mathfrak{M}}(n), \mathbb{Z})$ with geometric flavors will be constructed in $\$ 4$

Fix $n \geq 2$. Let $\mathcal{E}$ be a universal sheaf over $\overline{\mathfrak{M}}(n) \times X$, and let $\pi_{1}$ and $\pi_{2}$ be the two natural projections on $\overline{\mathfrak{M}}(n) \times X$. For $0 \leq k \leq 2$, define

$$
\mathcal{A}_{k}=R^{1} \pi_{1 *}\left(\mathcal{E} \otimes \pi_{2}^{*} \mathcal{O}_{X}(-k \ell)\right) .
$$

For $V \in \overline{\mathfrak{M}}(n)$, denote $V \otimes \mathcal{O}_{X}(k \ell)$ by $V \otimes \mathcal{O}_{X}(k)$ or $V(k)$. Then,

$$
\begin{gathered}
h^{0}(X, V(-k))=h^{2}(X, V(-k))=0 \quad \text { for } 0 \leq k \leq 2, \\
h^{1}(X, V)=h^{1}(X, V(-2))=n-1, \quad h^{1}(X, V(-1))=n
\end{gathered}
$$

by Proposition 1.5 in $\underline{\operatorname{Str}}$. It follows that the three sheaves $\mathcal{A}_{0}, \mathcal{A}_{1}, \mathcal{A}_{2}$ over $\overline{\mathfrak{M}}(n)$ are locally free of rank $n-1, n, n-1$ respectively.

Definition 3.1. Let $0 \leq k \leq 2$ and $r_{k}$ be the rank of $\mathcal{A}_{k}$. Define

$$
\begin{aligned}
\epsilon & =c_{1}\left(\mathcal{A}_{0}\right)-c_{1}\left(\mathcal{A}_{2}\right), \\
\delta & =n \cdot c_{1}\left(\mathcal{A}_{0}\right)-(n-1) \cdot c_{1}\left(\mathcal{A}_{1}\right), \\
\tau_{k} & =2 r_{k} \cdot c_{2}\left(\mathcal{A}_{k}\right)-\left(r_{k}-1\right) \cdot c_{1}\left(\mathcal{A}_{k}\right)^{2} .
\end{aligned}
$$

Note that these classes $\epsilon, \delta, \tau_{k}$ are independent of the choices of the universal sheaf $\mathcal{E}$ over $\overline{\mathfrak{M}}(n) \times X$. Let $K_{\overline{\mathfrak{M}}(n)}$ be the canonical class of $\overline{\mathfrak{M}}(n), \mathfrak{M}(n)$ be the open subset of $\overline{\mathfrak{M}}(n)$ parametrizing stable bundles, and

$$
\mathfrak{B}=\overline{\mathfrak{M}}(n)-\mathfrak{M}(n) .
$$

By the theorem in $\underline{\operatorname{Str}}, \operatorname{Pic}(\overline{\mathfrak{M}}(n))$ is freely generated by $\epsilon$ and $\delta$, and

$$
K_{\overline{\mathfrak{M}}(n)}=-3 \epsilon, \quad \mathfrak{B}=n \epsilon-2 \delta .
$$

Also, $a \epsilon+b \delta$ is ample if and only if $a, b>0$, and $\mathfrak{B}$ is irreducible and reduced.

It is known from [Bea, E-S, Mar] that the cohomology ring $H^{*}(\overline{\mathfrak{M}}(n) ; \mathbb{Z})$ is generated by the Chern classes of the bundles $\mathcal{A}_{0}, \mathcal{A}_{1}, \mathcal{A}_{2}$. It follows that $H^{4}(\overline{\mathfrak{M}}(n) ; \mathbb{Z})$ is the $\mathbb{Z}$-linear span of the six integral classes:

$$
\epsilon^{2}, \epsilon \delta, \delta^{2}, \tau_{0}, \tau_{1}, \tau_{2}
$$

By (3.1), the rank of $H^{4}(\overline{\mathfrak{M}}(n) ; \mathbb{Z})$ is 3 when $n=2$, and is 6 when $n \geq 3$. Therefore, if $n \geq 3$, then a linear basis of $H^{4}(\overline{\mathfrak{M}}(n) ; \mathbb{Z})$ is given by the six classes in (3.6). 
Remark 3.2. When $n=2$, the rank of $H_{4}(\overline{\mathfrak{M}}(n) ; \mathbb{Z})$ is different from that of the case $n \geq 3$. Therefore the construction of a basis of $H_{4}(\overline{\mathfrak{M}}(n) ; \mathbb{Z})$ in $\$$ for $n \geq 3$ needs to be modified. However, there is a method to describe the moduli space $\overline{\mathfrak{M}}(2)$ using the moduli spaces of stable sheaves on the Hirzebruch surface $\mathbb{F}_{1}$ and chamber structures, which enable us to compute all the Gromov-Witten invariants of $\overline{\mathfrak{M}}(2)$ (instead of a special kind considered in this paper for $n \geq 3$ ). Since the method is different, the result for $n=2$ will appear elsewhere.

\subsection{The boundary and the Uhlenbeck compactification.}

The quasi-projective variety $\mathfrak{M}(n)$ has a Uhlenbeck compactification

$$
\overline{\mathfrak{U}}(n)=\coprod_{0 \leq i \leq n-1} \mathfrak{M}(n-i) \times \operatorname{Sym}^{i}(X)
$$

according to [Uhl, LJ1, Mor]. Moreover, there exists a birational morphism, called the Gieseker-Uhlenbeck morphism,

$$
\Psi: \overline{\mathfrak{M}}(n) \rightarrow \overline{\mathfrak{U}}(n)
$$

sending $V \in \overline{\mathfrak{M}}(n)$ to the pair $\left(V^{* *}, \eta\right)$, where $V^{* *}$ is the double-dual of $V$ and

$$
\eta=\sum_{x \in X} h^{0}\left(X,\left(V^{* *} / V\right)_{x}\right) x
$$

Then the boundary divisor $\mathfrak{B}$ in (3.4) is contracted by $\Psi$ to the codimension-2 subset $\coprod_{1 \leq i \leq n-1} \mathfrak{M}(n-i) \times \operatorname{Sym}^{i}(X)$ in $\overline{\mathfrak{U}}(n)$.

Let $\mathfrak{B}_{*}=\Psi^{-1}(\mathfrak{M}(n-1) \times X) \subset \overline{\mathfrak{M}}(n)$ which parametrizes all $V \in \overline{\mathfrak{M}}(n)$ sitting in exact sequences $0 \rightarrow V \rightarrow V_{1} \rightarrow \mathcal{O}_{x} \rightarrow 0$ for some bundle $V_{1} \in \mathfrak{M}(n-1)$ and some point $x \in X$. It is an open dense subset of the boundary divisor $\mathfrak{B}$.

To construct a universal sheaf over $\mathfrak{B}_{*} \times X$, let $\mathcal{E}_{n-1}^{0}$ be a universal sheaf over $\mathfrak{M}(n-1) \times X$. By Q-Z, $\mathfrak{B}_{*} \cong \mathbb{P}\left(\mathcal{E}_{n-1}^{0}\right)$. For simplicity, write $\mathfrak{B}_{*}=\mathbb{P}\left(\mathcal{E}_{n-1}^{0}\right)$. Let

$$
\pi: \mathfrak{B}_{*}=\mathbb{P}\left(\mathcal{E}_{n-1}^{0}\right) \rightarrow \mathfrak{M}(n-1) \times X
$$

be the natural projection. Let $\Delta_{X}$ be the diagonal of $X \times X$. Consider the obvious isomorphism $\alpha: \mathfrak{M}(n-1) \times \Delta_{X} \rightarrow \mathfrak{M}(n-1) \times X$. Then, we have the isomorphisms:

$$
\begin{aligned}
&\left(\pi \times \operatorname{Id}_{X}\right)^{-1}\left(\mathfrak{M}(n-1) \times \Delta_{X}\right) \cong \mathbb{P}\left(\alpha^{*} \mathcal{E}_{n-1}^{0}\right), \\
&\left.\tilde{\pi}^{*} \mathcal{E}_{n-1}^{0}\right|_{\left(\pi \times \operatorname{Id}_{X}\right)^{-1}\left(\mathfrak{M}(n-1) \times \Delta_{X}\right)} \cong \tilde{\alpha}^{*} \mathcal{E}_{n-1}^{0},
\end{aligned}
$$

where $\tilde{\pi}: \mathfrak{B}_{*} \times X \rightarrow \mathfrak{M}(n-1) \times X$ is the composition of $\pi \times \operatorname{Id}_{X}$ and the map $\mathfrak{M}(n-1) \times X \times X \rightarrow \mathfrak{M}(n-1) \times X$ which denotes the projection to the product of the first and third factors, and $\tilde{\alpha}: \mathbb{P}\left(\alpha^{*} \mathcal{E}_{n-1}^{0}\right) \rightarrow \mathfrak{M}(n-1) \times X$ is the composition of the natural projection $\mathbb{P}\left(\alpha^{*} \mathcal{E}_{n-1}^{0}\right) \rightarrow \mathfrak{M}(n-1) \times \Delta_{X}$ and $\alpha$. A universal sheaf $\mathcal{E}^{\prime}$ over $\mathfrak{B}_{*} \times X$ sits in the exact sequence

$$
0 \rightarrow \mathcal{E}^{\prime} \rightarrow \tilde{\pi}^{*} \mathcal{E}_{n-1}^{0} \rightarrow \mathcal{O}_{\left(\pi \times \operatorname{Id}_{X}\right)^{-1}\left(\mathfrak{M}(n-1) \times \Delta_{X}\right)}(1) \rightarrow 0 .
$$

The following lemma will be used in later sections.

Lemma 3.3. Let $\tilde{\pi}_{i}$ be the $i$-th projection on $\mathfrak{M}(n-1) \times X$, and let

$$
\mathfrak{D}_{n-1}=2 c_{1}\left(\mathcal{A}_{n-1,1}^{0}\right)-c_{1}\left(\mathcal{A}_{n-1,2}^{0}\right)-c_{1}\left(\mathcal{A}_{n-1,0}^{0}\right),
$$

where $\mathcal{A}_{n-1, k}^{0}=R^{1} \tilde{\pi}_{1 *}\left(\mathcal{E}_{n-1}^{0} \otimes \tilde{\pi}_{2}^{*} \mathcal{O}_{X}(-k \ell)\right)$. Then, as divisors in $\mathfrak{B}_{*}$,

$$
\left.\mathfrak{B}_{*}\right|_{\mathfrak{B}_{*}}=-2 c_{1}\left(\mathcal{O}_{\mathfrak{B}_{*}}(1)\right)+\left(\tilde{\pi}_{1} \circ \pi\right)^{*} \mathfrak{D}_{n-1}+2\left(\tilde{\pi}_{2} \circ \pi\right)^{*} \ell .
$$


Proof. Since $\mathfrak{B}_{*}$ is open and dense in $\mathfrak{B}$, we see from (3.5) and Definition 3.1 that

$$
\left.\mathfrak{B}_{*}\right|_{\mathfrak{B}_{*}}=\left.\mathfrak{B}\right|_{\mathfrak{B}_{*}}=\left.\left(2(n-1) c_{1}\left(\mathcal{A}_{1}\right)-n c_{1}\left(\mathcal{A}_{2}\right)-n c_{1}\left(\mathcal{A}_{0}\right)\right)\right|_{\mathfrak{B}_{*}} .
$$

Next, let $0 \leq k \leq 2$, and let $\pi_{i}$ be the $i$-th projection on $\mathfrak{B}_{*} \times X$. Then the restriction of $\pi_{1}$ to the subset $\left(\pi \times \operatorname{Id}_{X}\right)^{-1}\left(\mathfrak{M}(n-1) \times \Delta_{X}\right)$ is an isomorphism from $\left(\pi \times \operatorname{Id}_{X}\right)^{-1}\left(\mathfrak{M}(n-1) \times \Delta_{X}\right)$ to $\mathfrak{B}_{*}$. Thus tensoring (3.11) by $\pi_{2}^{*} \mathcal{O}_{X}(-k \ell)$ and then applying the functor $\pi_{1 *}$, we get the exact sequence

$$
\begin{gathered}
0 \rightarrow \mathcal{O}_{\mathfrak{B}_{*}}(1) \otimes\left(\tilde{\pi}_{2} \circ \pi\right)^{*} \mathcal{O}_{X}(-k \ell) \rightarrow R^{1} \pi_{1 *}\left(\mathcal{E}^{\prime} \otimes \pi_{2}^{*} \mathcal{O}_{X}(-k \ell)\right) \\
\rightarrow R^{1} \pi_{1 *}\left(\tilde{\pi}^{*} \mathcal{E}_{n-1}^{0} \otimes \pi_{2}^{*} \mathcal{O}_{X}(-k \ell)\right) \rightarrow 0 .
\end{gathered}
$$

Note that $\pi_{2}=\tilde{\pi}_{2} \circ \tilde{\pi}$. Also, $R^{1} \pi_{1 *} \tilde{\pi}^{*} \cong\left(\tilde{\pi}_{1} \circ \pi\right)^{*} R^{1} \tilde{\pi}_{1 *}$ via the trivial base change:

$$
\begin{array}{cccc}
\mathfrak{B}_{*} & \times X & \stackrel{\pi_{1}}{\longrightarrow} & \mathfrak{B}_{*} \\
\downarrow \tilde{\pi} & & \downarrow \tilde{\pi}_{1} \circ \pi \\
\mathfrak{M}(n-1) \times X & \stackrel{\tilde{\pi}_{1}}{\longrightarrow} & \mathfrak{M}(n-1) .
\end{array}
$$

Therefore, rewriting the $3 \mathrm{rd}$ term in the above exact sequence, we obtain

$$
\begin{gathered}
0 \rightarrow \mathcal{O}_{\mathfrak{B}_{*}}(1) \otimes\left(\tilde{\pi}_{2} \circ \pi\right)^{*} \mathcal{O}_{X}(-k \ell) \rightarrow R^{1} \pi_{1 *}\left(\mathcal{E}^{\prime} \otimes \pi_{2}^{*} \mathcal{O}_{X}(-k \ell)\right) \\
\rightarrow\left(\tilde{\pi}_{1} \circ \pi\right)^{*} \mathcal{A}_{n-1, k}^{0} \rightarrow 0 .
\end{gathered}
$$

So the first Chern class $c_{1}\left(R^{1} \pi_{1 *}\left(\mathcal{E}^{\prime} \otimes \pi_{2}^{*} \mathcal{O}_{X}(-k \ell)\right)\right)$ equals

$$
c_{1}\left(\mathcal{O}_{\mathfrak{B}_{*}}(1)\right)-k\left(\tilde{\pi}_{2} \circ \pi\right)^{*} \ell+\left(\tilde{\pi}_{1} \circ \pi\right)^{*} c_{1}\left(\mathcal{A}_{n-1, k}^{0}\right),
$$

and the second Chern class $c_{2}\left(R^{1} \pi_{1 *}\left(\mathcal{E}^{\prime} \otimes \pi_{2}^{*} \mathcal{O}_{X}(-k \ell)\right)\right)$ is equal to

$$
\left(c_{1}\left(\mathcal{O}_{\mathfrak{B}_{*}}(1)\right)-k\left(\tilde{\pi}_{2} \circ \pi\right)^{*} \ell\right) \cdot\left(\tilde{\pi}_{1} \circ \pi\right)^{*} c_{1}\left(\mathcal{A}_{n-1, k}^{0}\right)+\left(\tilde{\pi}_{1} \circ \pi\right)^{*} c_{2}\left(\mathcal{A}_{n-1, k}^{0}\right) .
$$

Finally, we conclude from (3.13) and (3.14) that

$$
\left.\mathfrak{B}_{*}\right|_{\mathfrak{B}_{*}}=-2 c_{1}\left(\mathcal{O}_{\mathfrak{B}_{*}}(1)\right)+2\left(\tilde{\pi}_{2} \circ \pi\right)^{*} \ell+\left(\tilde{\pi}_{1} \circ \pi\right)^{*} \mathfrak{D},
$$

where

$$
\mathfrak{D}=2(n-1) c_{1}\left(\mathcal{A}_{n-1,1}^{0}\right)-n c_{1}\left(\mathcal{A}_{n-1,2}^{0}\right)-n c_{1}\left(\mathcal{A}_{n-1,0}^{0}\right) .
$$

Let $\epsilon_{1}, \delta_{1} \in \operatorname{Pic}(\overline{\mathfrak{M}}(n-1))$ be the counterparts of $\epsilon, \delta \in \operatorname{Pic}(\overline{\mathfrak{M}}(n))$. By (3.5) and Definition 3.1.

$$
\begin{aligned}
0 & =\left.(\overline{\mathfrak{M}}(n-1)-\mathfrak{M}(n-1))\right|_{\mathfrak{M}(n-1)} \\
& =\left.\left[(n-1) \epsilon_{1}-2 \delta_{1}\right]\right|_{\mathfrak{M}(n-1)} \\
& =2(n-2) c_{1}\left(\mathcal{A}_{n-1,1}^{0}\right)-(n-1) c_{1}\left(\mathcal{A}_{n-1,2}^{0}\right)-(n-1) c_{1}\left(\mathcal{A}_{n-1,0}^{0}\right) .
\end{aligned}
$$

So $\left.\mathfrak{B}_{*}\right|_{\mathfrak{B}_{*}}=-2 c_{1}\left(\mathcal{O}_{\mathfrak{B}_{*}}(1)\right)+2\left(\tilde{\pi}_{2} \circ \pi\right)^{*} \ell+\left(\tilde{\pi}_{1} \circ \pi\right)^{*} \mathfrak{D}_{n-1}$.

\subsection{Curves in $\overline{\mathfrak{M}}(n)$.}

We shall construct two curves in the Gieseker moduli space $\overline{\mathfrak{M}}(n)$ which freely generate the homology group $H_{2}(\overline{\mathfrak{M}}(n), \mathbb{Z})$. One such curve is a fiber $\mathfrak{f}$ of the morphism $\pi$ from (3.9). The following is Lemma 3.2 in $\mathrm{Q}-\mathrm{Z}$.

Lemma 3.4. Let $N_{\mathfrak{f} \subset \overline{\mathfrak{M}}(n)}$ be the normal bundle of $\mathfrak{f}$ in $\overline{\mathfrak{M}}(n)$. Then,

(i) $\mathfrak{f} \cdot K_{\overline{\mathfrak{M}}(n)}=0$ and $\mathfrak{f} \cdot \mathfrak{B}=-2$;

(ii) $N_{\mathfrak{f} \subset \overline{\mathfrak{M}}(n)} \cong \mathcal{O}_{\mathfrak{f}}^{\oplus(4 n-6)} \oplus \mathcal{O}_{\mathfrak{f}}(-2)$;

(iii) $\left.T_{\overline{\mathfrak{M}}(n)}\right|_{\mathfrak{f}} \cong \mathcal{O}_{\mathfrak{f}}^{\oplus(4 n-6)} \oplus \mathcal{O}_{\mathfrak{f}}(-2) \oplus \mathcal{O}_{\mathfrak{f}}(2)$. 
Next, we shall construct the other curve. Let $n \geq 3$, and let $\xi$ consist of $n$ distinct points in general position in $X=\mathbb{P}^{2}$. If $V$ sits in a nontrivial extension

$$
0 \rightarrow \mathcal{O}_{X}(-1) \rightarrow V \rightarrow I_{\xi} \rightarrow 0,
$$

then $V$ is stable and hence $V \in \overline{\mathfrak{M}}(n)$. Moreover, since $H^{0}\left(X, I_{\xi} \otimes \mathcal{O}_{X}(1)\right)=0$, the injection $\mathcal{O}_{X}(-1) \rightarrow V$ is unique up to scalars. It follows that

$$
\mathfrak{E}_{n}=\mathbb{P}\left(\operatorname{Ext}^{1}\left(I_{\xi}, \mathcal{O}_{X}(-1)\right)\right) \cong \mathbb{P}^{n-1}
$$

can be regarded as the subset of $\overline{\mathfrak{M}}(n)$ parametrizing all the sheaves $V \in \overline{\mathfrak{M}}(n)$ sitting in nontrivial extensions (3.16). A universal sheaf $\mathcal{E}^{\prime}$ over $\mathfrak{E}_{n} \times X$ sits in

$$
0 \rightarrow \pi_{2}^{*} \mathcal{O}_{X}(-\ell) \rightarrow \mathcal{E}^{\prime} \rightarrow \pi_{1}^{*} \mathcal{O}_{\mathfrak{E}_{n}}(-1) \otimes \pi_{2}^{*} I_{\xi} \rightarrow 0 .
$$

Tensoring by $\pi_{2}^{*} \mathcal{O}_{X}(-k \ell)$ and applying $\pi_{1 *}$ lead to the exact sequence:

$0 \rightarrow R^{1} \pi_{1 *}\left(\mathcal{E}^{\prime} \otimes \pi_{2}^{*} \mathcal{O}_{X}(-k \ell)\right) \rightarrow \mathcal{O}_{\mathfrak{E}_{n}}(-1)^{\oplus h^{1}\left(X, I_{\xi}(-k)\right)} \rightarrow \mathcal{O}_{\mathfrak{E}_{n}}^{\oplus h^{2}\left(X, \mathcal{O}_{X}(-(k+1) \ell)\right)} \rightarrow 0$, where $0 \leq k \leq 2$. An easy computation gives rise to the following:

$$
\begin{aligned}
c_{1}\left(R^{1} \pi_{1 *} \mathcal{E}^{\prime}\right) & =-(n-1) \cdot c_{1}\left(\mathcal{O}_{\mathfrak{E}_{n}}(1)\right), \\
c_{1}\left(R^{1} \pi_{1 *}\left(\mathcal{E}^{\prime} \otimes \pi_{2}^{*} \mathcal{O}_{X}(-k \ell)\right)\right) & =-n \cdot c_{1}\left(\mathcal{O}_{\mathfrak{E}_{n}}(1)\right),
\end{aligned}
$$

where $k=1,2$. It follows immediately from Definition 3.1 that

$$
\left.\epsilon\right|_{\mathfrak{E}_{n}}=c_{1}\left(\mathcal{O}_{\mathfrak{E}_{n}}(1)\right),\left.\quad \delta\right|_{\mathfrak{E}_{n}}=0 .
$$

Lemma 3.5. Let $n \geq 3$ and $\mathfrak{l}$ be a line in the projective space $\mathfrak{E}_{n}$.

(i) The homology group $H_{2}(\overline{\mathfrak{M}}(n) ; \mathbb{Z})$ is freely generated by $\mathfrak{f}$ and $\mathfrak{l}$.

(ii) The class $a \mathfrak{f}+b \mathfrak{l} \in H_{2}(\overline{\mathfrak{M}}(n) ; \mathbb{Z})$ is effective if and only if $a, b \geq 0$.

(iii) If $C \subset \overline{\mathfrak{M}}(n)$ is an irreducible curve contracted by the Gieseker-Uhlenbeck morphism $\Psi$, then $C=d \mathfrak{f} \in H_{2}(\overline{\mathfrak{M}}(n) ; \mathbb{Z})$ for some positive integer $d$.

Proof. (i) Since $\overline{\mathfrak{M}}(n)$ is rational, $H^{i}\left(\overline{\mathfrak{M}}(n), \mathcal{O}_{\overline{\mathfrak{M}}(n)}\right)=0$ for all $i \geq 1$. Hence $H^{2}(\overline{\mathfrak{M}}(n) ; \mathbb{Z}) \cong \operatorname{Pic}(\overline{\mathfrak{M}}(n))$, and $H^{2}(\overline{\mathfrak{M}}(n) ; \mathbb{Z})$ is freely generated by $\epsilon$ and $\delta$. By (3.19), $\mathfrak{l} \cdot \epsilon=1$ and $\mathfrak{l} \cdot \delta=0$. By Definition 3.1 and (3.14), $\mathfrak{f} \cdot \epsilon=0$ and $\mathfrak{f} \cdot \delta=1$. Since $H_{2}(\overline{\mathfrak{M}}(n) ; \mathbb{Z})$ is torsion-free, $H_{2}(\overline{\mathfrak{M}}(n) ; \mathbb{Z})$ is freely generated by $\mathfrak{f}$ and $\mathfrak{l}$.

(ii) Since $\mathfrak{f}$ and $\mathfrak{l}$ are effective, $a \mathfrak{f}+b \mathfrak{l}$ is effective if $a, b \geq 0$. Conversely, if $a \mathfrak{f}+b \mathfrak{l}$ is effective, then $a, b \geq 0$ since the divisor $c \epsilon+d \delta$ is ample if and only if $c, d>0$.

(iii) By (ii), $C=d \mathfrak{f}+b \mathfrak{l} \in H_{2}(\overline{\mathfrak{M}}(n) ; \mathbb{Z})$, where $d$ and $b$ are nonnegative integers not both zero. Let $L$ be a very ample divisor on $\overline{\mathfrak{U}}(n)$. Then, $\mathfrak{f} \cdot \Psi^{*} L=0$ and $\mathfrak{l} \cdot \Psi^{*} L \geq 0$. Since $\Psi^{*} L$ is a nonzero divisor and $H_{2}(\overline{\mathfrak{M}}(n) ; \mathbb{Z})$ is freely generated by $\mathfrak{f}$ and $\mathfrak{l}$, we must have $\mathfrak{l} \cdot \Psi^{*} L>0$. Thus, $C \cdot \Psi^{*} L=0$ forces $b=0$.

\section{A BASIS OF $H_{4}(\overline{\mathfrak{M}}(n) ; \mathbb{C})$}

In this section, we assume $n \geq 3$. Then the integral homology group $H_{4}(\overline{\mathfrak{M}}(n) ; \mathbb{Z})$ is free of rank 6 . In the following, we construct a basis $\left\{\Xi_{1}, \ldots, \Xi_{6}\right\}$ for $H_{4}(\overline{\mathfrak{M}}(n) ; \mathbb{C})$. This construction makes use of a result due to Hirschowitz and Hulek.

We review the results in $[\mathrm{H}-\mathrm{H}]$, where complete rational curves were found in $\mathfrak{M}(n)$. Let $n \geq 2$ and $\Gamma=\mathbb{P}^{1}$. Fix lines $\ell_{1}, \ldots, \ell_{n} \subset X=\mathbb{P}^{2}$ in general position. For $1 \leq i \leq n$, let $\phi_{i}: \Gamma \rightarrow \ell_{i}$ be an isomorphism, and define $Y_{i} \subset \Gamma \times X$ to be the graph of $\phi_{i}$. For generic choices of $\phi_{1}, \ldots, \phi_{n}$, it was proved in $[\mathrm{H}-\mathrm{H}]$ that $Y_{1}, \ldots, Y_{n}$ 
are disjoint. Moreover, if $N_{Y / \Gamma \times X}$ denotes the normal bundle of $Y \stackrel{\text { def }}{=} \coprod_{i=1}^{n} Y_{i}$ in $\Gamma \times X$, then $\left.\operatorname{det}\left(N_{Y / \Gamma \times X}\right) \cong \mathcal{O}_{\Gamma \times X}(2,1)\right|_{Y}$. Therefore, the element

$$
1 \in H^{0}\left(Y, \mathcal{O}_{Y}\right) \cong \operatorname{Ext}^{1}\left(\mathcal{O}_{\Gamma \times X}(2,0) \otimes I_{Y}, \mathcal{O}_{\Gamma \times X}(0,-1)\right)
$$

defines a rank-2 bundle $\widetilde{\mathcal{E}}_{n}$ over $\Gamma \times X$ sitting in an exact sequence

$$
0 \rightarrow \mathcal{O}_{\Gamma \times X}(0,-1) \rightarrow \widetilde{\mathcal{E}}_{n} \rightarrow \mathcal{O}_{\Gamma \times X}(2,0) \otimes I_{Y} \rightarrow 0
$$

such that $\left.\widetilde{\mathcal{E}}_{n}\right|_{\{p\} \times X} \in \mathfrak{M}(n)$ for all $p \in \Gamma$, and $\widetilde{\mathcal{E}}_{n}$ induces a nonconstant morphism

$$
\iota: \Gamma \rightarrow \mathfrak{M}(n) \text {. }
$$

Let $\tilde{\pi}_{1}$ and $\tilde{\pi}_{2}$ be the natural projections on $\Gamma \times X$. Let

$$
\widetilde{\mathcal{A}}_{n, k}=R^{1} \tilde{\pi}_{1 *}\left(\widetilde{\mathcal{E}}_{n} \otimes \tilde{\pi}_{2}^{*} \mathcal{O}_{X}(-k \ell)\right) .
$$

By Lemma 3.5 in $[\mathrm{H}-\mathrm{H}]$, the degrees of the bundles $\widetilde{\mathcal{A}}_{n, k}$ are

$$
a_{n, k}= \begin{cases}2 n-2 & \text { if } k=0 \\ n & \text { if } k=1 \\ 0 & \text { if } k=2\end{cases}
$$

Remark 4.1. (i) Let $n \geq 2$. By (3.3), the condition in Corollarie (6.9.9) of [Gro] is satisfied. Hence the base-change theorem of the first direct image holds for every projective morphism to $\overline{\mathfrak{M}}(n)$ and for the sheaf $\mathcal{E} \otimes \pi_{2}^{*} \mathcal{O}_{X}(-k \ell)$ with $0 \leq k \leq 2$. For a finite morphism $\varphi$ from $Y$ onto a subvariety of $\overline{\mathfrak{M}}(n)$, the intersection numbers of $\varphi(Y)$ with $\epsilon^{2}, \epsilon \cdot \delta, \delta^{2}, \tau_{0}, \tau_{1}, \tau_{2}$ can be computed on $Y$, via the projection formula, by pulling back the Chern classes of the first direct images of the sheaf $\mathcal{E} \otimes \pi_{2}^{*} \mathcal{O}_{X}(-k \ell)$ with $0 \leq k \leq 2$.

(ii) Let $n \geq 3$. Then $(n-1) \geq 2$. In Subsection 4.2, we will construct a surface $Y=\pi^{-1}(\Gamma \times\{y\})$ admitting a finite morphism onto a surface $\Xi_{3}$ of $\overline{\mathfrak{M}}(n)$. This finite morphism factors through the nonconstant morphism $\iota: \Gamma \rightarrow \mathfrak{M}(n-1)$. Applying (i), we may assume for convenience that $\Gamma \subset \mathfrak{M}(n-1)$ and consequently $Y=\Xi_{3} \subset \overline{\mathfrak{M}}(n)$ (in fact, it can be proved that when $n \geq 5$, the morphism $\iota: \Gamma \rightarrow \mathfrak{M}(n-1)$ is injective). A similar discussion works for $\Xi_{4}$.

4.1. The homology classes $\Xi_{1}, \Xi_{2} \in H_{4}(\overline{\mathfrak{M}}(n) ; \mathbb{C})$. Let $n \geq 3$, and assume $\Gamma \subset$ $\mathfrak{M}(n-1)$ as pointed out in Remark 4.1 (ii). Let $\mathbb{P}=\mathbb{P}\left(\widetilde{\mathcal{E}}_{n-1}\right)=\pi^{-1}(\Gamma \times X) \subset \mathfrak{B}_{*}$. Then $\mathbb{P}$ parametrizes all the sheaves $V \in \overline{\mathfrak{M}}(n)$ sitting in

$$
\left.0 \rightarrow V \rightarrow \widetilde{\mathcal{E}}_{n-1}\right|_{\{p\} \times X} \rightarrow \mathcal{O}_{x} \rightarrow 0
$$

for some $p \in \Gamma$ and $x \in X$. We still use $\pi$ to denote the natural projection

$$
\pi: \mathbb{P} \rightarrow \Gamma \times X
$$

By (3.11), a universal sheaf $\widetilde{\mathcal{E}}$ over $\mathbb{P} \times X$ sits in the exact sequence

$$
0 \rightarrow \widetilde{\mathcal{E}} \rightarrow \tilde{\pi}^{*} \widetilde{\mathcal{E}}_{n-1} \rightarrow \mathcal{O}_{\left(\pi \times \operatorname{Id}_{X}\right)^{-1}\left(\mathbb{P} \times \Delta_{X}\right)}(1) \rightarrow 0,
$$

where $\tilde{\pi}$ is the composition of $\pi \times \operatorname{Id}_{X}: \mathbb{P} \times X \rightarrow \Gamma \times X \times X$ and the projection $\Gamma \times X \times X \rightarrow \Gamma \times X$ to the first and third factors.

Let $\pi_{i}$ (respectively, $\tilde{\pi}_{i}$ ) be the natural projections on $\mathbb{P} \times X$ (respectively, $\left.\Gamma \times X\right)$. By (3.14) and (3.15),$c_{1}\left(R^{1} \pi_{1 *}\left(\widetilde{\mathcal{E}} \otimes \pi_{2}^{*} \mathcal{O}_{X}(-k \ell)\right)\right)$ equals

$$
c_{1}\left(\mathcal{O}_{\mathbb{P}}(1)\right)-k\left(\tilde{\pi}_{2} \circ \pi\right)^{*} \ell+\left(\tilde{\pi}_{1} \circ \pi\right)^{*} c_{1}\left(\widetilde{\mathcal{A}}_{n-1, k}\right),
$$


where $\widetilde{\mathcal{A}}_{n-1, k}$ is defined in (4.2), and $c_{2}\left(R^{1} \pi_{1 *}\left(\widetilde{\mathcal{E}} \otimes \pi_{2}^{*} \mathcal{O}_{X}(-k \ell)\right)\right)$ equals

$$
\left(c_{1}\left(\mathcal{O}_{\mathbb{P}}(1)\right)-k\left(\tilde{\pi}_{2} \circ \pi\right)^{*} \ell\right) \cdot\left(\tilde{\pi}_{1} \circ \pi\right)^{*} c_{1}\left(\widetilde{\mathcal{A}}_{n-1, k}\right) .
$$

In addition, we conclude from (4.1) that

$$
\begin{aligned}
c_{1}\left(\mathcal{O}_{\mathbb{P}}(1)\right)^{2} & =\pi^{*} c_{1}\left(\widetilde{\mathcal{E}}_{n-1}\right) \cdot c_{1}\left(\mathcal{O}_{\mathbb{P}}(1)\right)-\pi^{*} c_{2}\left(\widetilde{\mathcal{E}}_{n-1}\right) \\
& =\pi^{*}(2,-1) \cdot c_{1}\left(\mathcal{O}_{\mathbb{P}}(1)\right)-\pi^{*}\left[(2,0) \cdot(0,-1)+\sum_{i=1}^{n-1} Y_{i}\right] .
\end{aligned}
$$

Fix a point $p \in \Gamma$ and let $V_{1}=\left.\widetilde{\mathcal{E}}_{n-1}\right|_{\{p\} \times X}$. Consider $\mathbb{P}\left(V_{1}\right) \subset \mathbb{P} \subset \overline{\mathfrak{M}}(n)$. By Definition 3.1, (4.5), (4.6) and (4.7), we obtain

$$
\begin{aligned}
\left.\epsilon\right|_{\mathbb{P}\left(V_{1}\right)} & =2 \pi^{*} \ell, \\
\left.\delta\right|_{\mathbb{P}\left(V_{1}\right)} & =c_{1}\left(\mathcal{O}_{\mathbb{P}\left(V_{1}\right)}(1)\right)+(n-1) \pi^{*} \ell, \\
\left.\tau_{k}\right|_{\mathbb{P}\left(V_{1}\right)} & =-\left(r_{k}-1\right)\left(c_{1}\left(\mathcal{O}_{\mathbb{P}\left(V_{1}\right)}(1)\right)-k \pi^{*} \ell\right)^{2}, \\
c_{1}\left(\mathcal{O}_{\mathbb{P}\left(V_{1}\right)}(1)\right)^{2} & =-\pi^{*} \ell \cdot c_{1}\left(\mathcal{O}_{\mathbb{P}\left(V_{1}\right)}(1)\right)-(n-1) \pi^{*} x,
\end{aligned}
$$

where by abusing notation, $\pi$ denotes the natural projection $\mathbb{P}\left(V_{1}\right) \rightarrow X$.

Define $\Xi_{1} \in H_{4}(\overline{\mathfrak{M}}(n) ; \mathbb{C})$ to be the surface $\pi^{-1}(\{p\} \times \ell) \subset \mathbb{P}\left(V_{1}\right) \subset \mathbb{P} \subset \overline{\mathfrak{M}}(n)$, where $\ell \subset X=\mathbb{P}^{2}$ is a fixed line. Then we have

$$
\begin{array}{cc}
\epsilon^{2} \cdot \Xi_{1}=0, \quad \epsilon \cdot \delta \cdot \Xi_{1}=2, \quad \delta^{2} \cdot \Xi_{1}=2 n-3, \\
\tau_{0} \cdot \Xi_{1}=n-2, \quad \tau_{1} \cdot \Xi_{1}=3 n-3, \quad \tau_{2} \cdot \Xi_{1}=5 n-10 .
\end{array}
$$

Next, define $\Xi_{2} \in H_{4}(\overline{\mathfrak{M}}(n) ; \mathbb{C})$ to be $c_{1}\left(\mathcal{O}_{\mathbb{P}\left(V_{1}\right)}(1)\right)$ regarded as a 2-dimensional cycle in $\overline{\mathfrak{M}}(n)$ via the inclusion $\mathbb{P}\left(V_{1}\right) \subset \overline{\mathfrak{M}}(n)$. Then

$$
\begin{aligned}
\epsilon^{2} \cdot \Xi_{2}=4, \quad \epsilon \cdot \delta \cdot \Xi_{2} & =2 n-4, \quad \delta^{2} \cdot \Xi_{2}=n^{2}-5 n+5, \\
\tau_{0} \cdot \Xi_{2} & =(n-2)^{2}, \tau_{1} \cdot \Xi_{2}=(n-1)(n-5), \tau_{2} \cdot \Xi_{2}=(n-2)(n-10) .
\end{aligned}
$$

4.2. The homology classes $\Xi_{3}, \Xi_{4} \in H_{4}(\overline{\mathfrak{M}}(n) ; \mathbb{C})$.

Fix a line $\ell \subset X=\mathbb{P}^{2}$. Let $W=\pi^{-1}(\Gamma \times \ell) \subset \mathbb{P} \subset \overline{\mathfrak{M}}(n)$, where $\Gamma, \mathbb{P}$ and $\pi$ are from the previous subsection. By Definition 3.1, (4.3), (4.5) and (4.6),

$$
\begin{aligned}
\left.\epsilon\right|_{W}= & \pi^{*}(2 n-4,2) \\
\left.\delta\right|_{W}= & c_{1}\left(\mathcal{O}_{W}(1)\right)+\pi^{*}\left(n^{2}-2 n-1, n-1\right), \\
\left.\tau_{k}\right|_{W}= & \pi^{*}\left(2 a_{n-1, k}-2\left(r_{k}-1\right),(2 k+1)\left(r_{k}-1\right)\right) \cdot c_{1}\left(\mathcal{O}_{W}(1)\right) \\
& \quad+\left(\left(r_{k}-1\right)(n-3)-2 k a_{n-1, k}\right) \pi^{*} x
\end{aligned}
$$

where $x \in \Gamma \times \ell$ is a fixed point and by abusing notation, $\pi: W \rightarrow \Gamma \times \ell \cong \mathbb{P}^{1} \times \mathbb{P}^{1}$ stands for the natural projection. In addition, by (4.7), we conclude that

$$
c_{1}\left(\mathcal{O}_{W}(1)\right)^{2}=\pi^{*}(2,-1) c_{1}\left(\mathcal{O}_{W}(1)\right)-(n-3) \pi^{*} x .
$$

Define $\Xi_{3} \in H_{4}(\overline{\mathfrak{M}}(n) ; \mathbb{C})$ to be the surface $\pi^{-1}(\Gamma \times\{y\}) \subset W \subset \overline{\mathfrak{M}}(n)$, where $y \in \ell$ is a fixed point. A straightforward computation shows that

$$
\begin{gathered}
\epsilon^{2} \cdot \Xi_{3}=0, \quad \epsilon \cdot \delta \cdot \Xi_{3}=2 n-4, \quad \delta^{2} \cdot \Xi_{3}=2 n^{2}-4 n, \\
\tau_{0} \cdot \Xi_{3}=2 n-4, \quad \tau_{1} \cdot \Xi_{3}=0, \quad \tau_{2} \cdot \Xi_{3}=-2 n+4 .
\end{gathered}
$$


Define $\Xi_{4} \in H_{4}(\overline{\mathfrak{M}}(n) ; \mathbb{C})$ to be $c_{1}\left(\mathcal{O}_{W}(1)\right)$ regarded as a 2-dimensional cycle in $\overline{\mathfrak{M}}(n)$ via the inclusion $W \subset \overline{\mathfrak{M}}(n)$. Then we have

$$
\epsilon^{2} \cdot \Xi_{4}=8(n-2), \epsilon \cdot \delta \cdot \Xi_{4}=4 n^{2}-12 n+10, \delta^{2} \cdot \Xi_{4}=2 n^{3}-8 n^{2}+9 n-1,
$$

$$
\tau_{0} \cdot \Xi_{4}=n^{2}-5 n+6, \tau_{1} \cdot \Xi_{4}=n^{2}-1, \tau_{2} \cdot \Xi_{4}=(n-2)(n+9) .
$$

4.3. The homology class $\Xi_{5} \in H_{4}(\overline{\mathfrak{M}}(n) ; \mathbb{C})$.

Since $n \geq 3$, the moduli space $\mathfrak{M}(n-2)$ is nonempty. Fix a vector bundle $V_{2} \in \mathfrak{M}(n-2)$ and two distinct points $x_{1}, x_{2} \in X$. Let $\Xi_{5} \subset \overline{\mathfrak{M}}(n)$ parametrize all the sheaves $V \in \overline{\mathfrak{M}}(n)$ sitting in exact sequences:

$$
0 \rightarrow V \rightarrow V_{2} \rightarrow \mathcal{O}_{x_{1}} \oplus \mathcal{O}_{x_{2}} \rightarrow 0
$$

Then we have the isomorphisms $\Xi_{5} \cong \mathbb{P}\left(\left.V\right|_{x_{1}}\right) \times \mathbb{P}\left(\left.V\right|_{x_{2}}\right) \cong \mathbb{P}^{1} \times \mathbb{P}^{1}$. Moreover, a universal sheaf $\mathcal{E}^{\prime}$ over $\Xi_{5} \times X$ sits in the exact sequence:

$$
\left.\left.0 \rightarrow \mathcal{E}^{\prime} \rightarrow \pi_{2}^{*} V_{2} \rightarrow \tilde{\pi}_{1}^{*} \mathcal{O}_{\mathbb{P}^{1}}(1)\right|_{\mathbb{P}^{1} \times \mathbb{P}^{1} \times\left\{x_{1}\right\}} \oplus \tilde{\pi}_{2}^{*} \mathcal{O}_{\mathbb{P}^{1}}(1)\right|_{\mathbb{P}^{1} \times \mathbb{P}^{1} \times\left\{x_{2}\right\}} \rightarrow 0,
$$

where $\pi_{i}$ and $\tilde{\pi}_{i}$ denote the $i$-th projection on $\Xi_{5} \times X$ and $\mathbb{P}^{1} \times \mathbb{P}^{1} \times X$, respectively. Tensoring the above exact sequence by $\left.\pi_{2}^{*} \mathcal{O}_{X}(-k \ell)\right)$ and applying $\pi_{1 *}$ yield

$$
0 \rightarrow \mathcal{O}_{\Xi_{5}}(1,0) \oplus \mathcal{O}_{\Xi_{5}}(0,1) \rightarrow R^{1} \pi_{1 *}\left(\mathcal{E}^{\prime} \otimes \pi_{2}^{*} \mathcal{O}_{X}(-k \ell)\right) \rightarrow \mathcal{O}_{\Xi_{5}}^{\oplus h^{1}\left(X, V_{2}(-k)\right)} \rightarrow 0,
$$

where $0 \leq k \leq 2$. Therefore $c_{1}\left(R^{1} \pi_{1 *}\left(\mathcal{E}^{\prime} \otimes \pi_{2}^{*} \mathcal{O}_{X}(-k \ell)\right)\right)=c_{1}\left(\mathcal{O}_{\Xi_{5}}(1,1)\right)$ and $c_{2}\left(R^{1} \pi_{1 *}\left(\mathcal{E}^{\prime} \otimes \pi_{2}^{*} \mathcal{O}_{X}(-k \ell)\right)\right)=1$ for $0 \leq k \leq 2$. It follows from Definition 3.1 that

$$
\left.\epsilon\right|_{\Xi_{5}}=0,\left.\quad \delta\right|_{\Xi_{5}}=c_{1}\left(\mathcal{O}_{\Xi_{5}}(1,1)\right) .
$$

Regarding $\Xi_{5} \in H_{4}(\overline{\mathfrak{M}}(n) ; \mathbb{C})$, we obtain the intersection numbers on $\overline{\mathfrak{M}}(n)$ :

$$
\epsilon^{2} \cdot \Xi_{5}=\epsilon \cdot \delta \cdot \Xi_{5}=0, \quad \delta^{2} \cdot \Xi_{5}=2, \tau_{0} \cdot \Xi_{5}=\tau_{1} \cdot \Xi_{5}=\tau_{2} \cdot \Xi_{5}=2 .
$$

4.4. The surface $\Xi_{6}$ in $\overline{\mathfrak{M}}(n)$.

Fix a vector bundle $V_{2} \in \mathfrak{M}(n-2)$ and a point $x \subset X$. Fix a trivialization of $V_{2}$ in an open neighborhood $O_{x}$ of $x$. Let $X^{[2]}$ be the Hilbert scheme parametrizing the length-2 closed subschemes of $X$, and let

$$
M_{2}(x)=\left\{\xi \in X^{[2]} \mid \operatorname{Supp}(\xi)=\{x\}\right\} \cong \mathbb{P}^{1} .
$$

For $\xi \in M_{2}(x)$, let $\iota_{\xi}: \mathcal{O}_{X} \rightarrow \mathcal{O}_{\xi}$ be the natural quotient morphism.

Let $\Xi_{6}=\mathbb{P}^{1} \times M_{2}(x)$ be the subset of $\overline{\mathfrak{M}}(n)$ parametrizing all the sheaves $V \in \overline{\mathfrak{M}}(n)$ sitting in extensions of the form

$$
0 \longrightarrow V \longrightarrow V_{2} \stackrel{(a, b)}{\longrightarrow} \mathcal{O}_{\xi} \longrightarrow 0
$$

where $\xi \in M_{2}(x)$, and for $(a, b) \in \mathbb{P}^{1}$, the map $V_{2} \stackrel{(a, b)}{\longrightarrow} \mathcal{O}_{\xi}$ denotes the composition:

$$
\left.V_{2} \longrightarrow V_{2}\right|_{O_{x}} \cong \mathcal{O}_{O_{x}}^{\oplus 2} \stackrel{\left(a \iota_{\xi}, b \iota_{\xi}\right)}{\longrightarrow} \mathcal{O}_{\xi} .
$$

Next, we construct a universal sheaf over $\Xi_{6} \times X=\mathbb{P}^{1} \times M_{2}(x) \times X$. Let $\pi_{i_{1}, \ldots, i_{m}}$ denote the projection of $\mathbb{P}^{1} \times M_{2}(x) \times X$ to the product of the $i_{1}$-th, $\ldots$, $i_{m}$-th factors. Over $\Xi_{6}=\mathbb{P}^{1} \times M_{2}(x)$, there is a tautological surjection $\mathcal{O}_{\Xi_{6}}^{\oplus 2} \rightarrow$ $\mathcal{O}_{\Xi_{6}}(1,0) \rightarrow 0$. This pulls back to a surjection over $\mathbb{P}^{1} \times M_{2}(x) \times X$ :

$$
\mathcal{O}_{\Xi_{6} \times X}^{\oplus 2} \rightarrow \pi_{1}^{*} \mathcal{O}_{\mathbb{P}^{1}}(1) \rightarrow 0
$$


Let $\mathcal{Z}_{2}(x)$ be the universal codimension-2 subscheme in $M_{2}(x) \times X$, and let $\mathcal{O}_{M_{2}(x) \times X} \rightarrow \mathcal{O}_{\mathcal{Z}_{2}(x)} \rightarrow 0$ be the natural surjection. Pulling back to $\mathbb{P}^{1} \times M_{2}(x) \times X$ yields a surjection:

$$
\mathcal{O}_{\Xi_{6} \times X} \rightarrow \pi_{2,3}^{*} \mathcal{O}_{\mathcal{Z}_{2}(x)} \rightarrow 0
$$

Tensoring (4.15) and (4.16), we obtain a surjection

$$
\mathcal{O}_{\Xi_{6} \times X}^{\oplus 2} \rightarrow \pi_{1}^{*} \mathcal{O}_{\mathbb{P}^{1}}(1) \otimes \pi_{2,3}^{*} \mathcal{O}_{\mathcal{Z}_{2}(x)} \rightarrow 0 .
$$

Since $\mathcal{Z}_{2}(x)$ is supported on $M_{2}(x) \times\{x\}$, in view of the trivialization of $V_{2}$ near $x$, (4.17) induces a surjection $\pi_{3}^{*} V_{2} \rightarrow \pi_{1}^{*} \mathcal{O}_{\mathbb{P}^{1}}(1) \otimes \pi_{2,3}^{*} \mathcal{O}_{\mathcal{Z}_{2}(x)} \rightarrow 0$. The kernel $\mathcal{E}^{\prime}$ of the map $\pi_{3}^{*} V_{2} \rightarrow \pi_{1}^{*} \mathcal{O}_{\mathbb{P}^{1}}(1) \otimes \pi_{2,3}^{*} \mathcal{O}_{\mathcal{Z}_{2}(x)}$ is a universal sheaf over $\Xi_{6} \times X$ :

$$
0 \rightarrow \mathcal{E}^{\prime} \rightarrow \pi_{3}^{*} V_{2} \rightarrow \pi_{1}^{*} \mathcal{O}_{\mathbb{P}^{1}}(1) \otimes \pi_{2,3}^{*} \mathcal{O}_{\mathcal{Z}_{2}(x)} \rightarrow 0 .
$$

It follows that for $0 \leq k \leq 2$, we have the exact sequence over $\Xi_{6}$ :

$$
\begin{aligned}
0 \rightarrow \tilde{\pi}_{1}^{*} \mathcal{O}_{\mathbb{P}^{1}}(1) \otimes \tilde{\pi}_{2}^{*}\left(\left.\mathcal{O}_{X}^{[2]}\right|_{M_{2}(x)}\right) \rightarrow R^{1}\left(\pi_{1,2}\right)_{*}\left(\mathcal{E}^{\prime} \otimes \pi_{3}^{*} \mathcal{O}_{X}(-k \ell)\right) \\
\rightarrow \mathcal{O}_{\Xi_{6}}^{\oplus h^{1}\left(X, V_{2}(-k)\right)} \rightarrow 0,
\end{aligned}
$$

where $\tilde{\pi}_{1}$ and $\tilde{\pi}_{2}$ are the two natural projections on $\Xi_{6}=\mathbb{P}^{1} \times M_{2}(x)$, and $\mathcal{O}_{X}^{[2]}$ is the tautological rank-2 bundle over the Hilbert scheme $X^{[2]}$ whose fiber at a point $\xi \in X^{[2]}$ is the space $H^{0}\left(X, \mathcal{O}_{\xi}\right)$. It is well known that $-2 c_{1}\left(\mathcal{O}_{X}^{[2]}\right)=M_{2}(X)$, where $M_{2}(X) \subset X^{[2]}$ consists of all the elements $\xi \in X^{[2]}$ such that $|\operatorname{Supp}(\xi)|=1$. Since $M_{2}(X) \cdot M_{2}(x)=-2$, we conclude from the above exact sequence that

$$
\begin{aligned}
& c_{1}\left(R^{1}\left(\pi_{1,2}\right)_{*}\left(\mathcal{E}^{\prime} \otimes \pi_{3}^{*} \mathcal{O}_{X}(-k \ell)\right)\right)=(2,1) \in \operatorname{Pic}\left(\Xi_{6}\right) \cong \operatorname{Pic}\left(\mathbb{P}^{1} \times \mathbb{P}^{1}\right), \\
& c_{2}\left(R^{1}\left(\pi_{1,2}\right)_{*}\left(\mathcal{E}^{\prime} \otimes \pi_{3}^{*} \mathcal{O}_{X}(-k \ell)\right)\right)=1 .
\end{aligned}
$$

By Definition 3.1 we get the six intersection numbers on $\overline{\mathfrak{M}}(n)$ :

$$
\begin{gathered}
\epsilon^{2} \cdot \Xi_{6}=0, \quad \epsilon \cdot \delta \cdot \Xi_{6}=0, \quad \delta^{2} \cdot \Xi_{6}=4, \\
\tau_{0} \cdot \Xi_{6}=-2 n+6, \quad \tau_{1} \cdot \Xi_{6}=-2 n+4, \quad \tau_{2} \cdot \Xi_{6}=-2 n+6 .
\end{gathered}
$$

Now we can summarize the above in the following proposition.

Proposition 4.2. Let $n \geq 3$. Then $\left\{\Xi_{1}, \ldots, \Xi_{6}\right\}$ is a linear basis of $H_{4}(\overline{\mathfrak{M}}(n) ; \mathbb{C})$.

Proof. Note from (4.9), (4.1), (4.12), (4.13), (4.14) and (4.18) that the intersection matrix between the classes $\Xi_{1}, \ldots, \Xi_{6}$ and the classes $\epsilon^{2}, \epsilon \cdot \delta, \delta^{2}, \tau_{0}, \tau_{1}, \tau_{2}$ has a nonzero determinant. Since $H_{4}(\overline{\mathfrak{M}}(n) ; \mathbb{C})$ has dimension 6 , our result follows.

\section{The Restriction of the ObStruCtion SHEAF ON CERTAIN OPEN SUBSETS}

From the previous section, we see that, if we let $\overline{\mathfrak{M}}_{*}(n)=\mathfrak{B}_{*} \cup \mathfrak{M}(n)$, then the classes $\Xi_{1}, \Xi_{2}, \Xi_{3}, \Xi_{4}$ lie in $\overline{\mathfrak{M}}_{*}(n)$ while $\Xi_{5}$ and $\Xi_{6}$ lie in the complement $\overline{\mathfrak{M}}(n)-$ $\overline{\mathfrak{M}}_{*}(n)$. Since a stable map $[\mu:(D, p) \rightarrow \overline{\mathfrak{M}}(n)]$ in $e v_{1}^{-1}\left(\Xi_{i}\right) \subset \overline{\mathfrak{M}}_{0,1}(\overline{\mathfrak{M}}(n), d \mathfrak{f})$ has $\mu(p) \in \Xi_{i}$ for $1 \leq i \leq 4$, we have $\mu(D) \subset \mathfrak{B}_{*}$. In this section, we use the geometric construction of $\mathfrak{B}_{*}$ in Subsect. 3.2 to effectively compute the virtual cycle restricted to $e v_{1}^{-1}\left(\mathfrak{B}_{*}\right)$. The result will be used to compute the Gromov-Witten invariants $\langle\alpha\rangle_{0, d f}$ when $\alpha$ is dual to the classes $\Xi_{1}, \Xi_{2}, \Xi_{3}, \Xi_{4}$.

Fix $d \geq 1$. Consider the open subset $\mathfrak{O}_{0}$ of $\overline{\mathfrak{M}}_{0,0}(\overline{\mathfrak{M}}(n), d \mathfrak{f})$ consisting of stable maps $[\mu: D \rightarrow \overline{\mathfrak{M}}(n)]$ such that $\mu(D) \subset \overline{\mathfrak{M}}_{*}(n)$. Similarly, take the open subset $\mathfrak{O}_{1}$ 
of $\overline{\mathfrak{M}}_{0,1}(\overline{\mathfrak{M}}(n), d \mathfrak{f})$ consisting of stable maps $[\mu:(D ; p) \rightarrow \overline{\mathfrak{M}}(n)]$ such that $\mu(D) \subset$ $\overline{\mathfrak{M}}_{*}(n)$. Clearly $\mathfrak{O}_{1}=f_{1,0}^{-1}\left(\mathfrak{O}_{0}\right)$. Let $[\mu:(D ; p) \rightarrow \overline{\mathfrak{M}}(n)] \in \mathfrak{O}_{1}$. Since $\mu(D)=d \mathfrak{f}$ in $H_{2}(\overline{\mathfrak{M}}(n) ; \mathbb{Z}), \mu(D) \subset \mathfrak{B}_{*}$ and $\mu(D)$ is a fiber of the projection $\pi$. Moreover, the composition $\Psi \circ e v_{1}$ sends the stable map $[\mu:(D ; p) \rightarrow \overline{\mathfrak{M}}(n)]$ to a point in $\mathfrak{M}(n-1) \times X \subset \overline{\mathfrak{U}}(n)$, which is independent of the marked point $p$ on $D$. Hence $e v_{1}$ induces a morphism $\phi$ from $\mathfrak{O}_{0}$ to $\mathfrak{M}(n-1) \times X$. Putting $\widetilde{e v}_{1}=\left.e v_{1}\right|_{\mathfrak{O}_{1}}$ and $\tilde{f}_{1,0}=\left.f_{1,0}\right|_{\mathfrak{O}_{1}}$, we have the following commutative diagram:

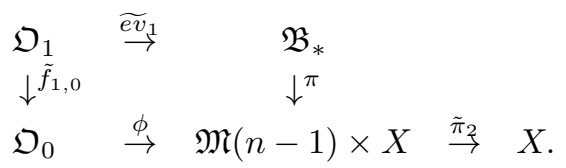

Note that the fiber $\phi^{-1}\left(V_{1}, x\right)$ over a point $\left(V_{1}, x\right) \in \mathfrak{M}(n-1) \times X$ is simply

$$
\overline{\mathfrak{M}}_{0,0}\left(\pi^{-1}\left(V_{1}, x\right), d\left[\pi^{-1}\left(V_{1}, x\right)\right]\right)
$$

which is isomorphic to $\overline{\mathfrak{M}}_{0,0}\left(\mathbb{P}^{1}, d\left[\mathbb{P}^{1}\right]\right)$ via the isomorphism $\pi^{-1}\left(V_{1}, x\right) \cong \mathbb{P}^{1}$. Hence the complex dimension of the open subset $\mathfrak{O}_{0} \subset \overline{\mathfrak{M}}_{0,0}(\overline{\mathfrak{M}}(n), d \mathfrak{f})$ is equal to

$$
\begin{aligned}
& \operatorname{dim} \overline{\mathfrak{M}}_{0,0}\left(\mathbb{P}^{1}, d\left[\mathbb{P}^{1}\right]\right)+\operatorname{dim} \mathfrak{M}(n-1)+\operatorname{dim} X \\
= & (2 d-2)+[4(n-1)-4]+2 \\
= & 2 d+4 n-8 .
\end{aligned}
$$

Since $K_{\overline{\mathfrak{M}}(n)} \cdot d \mathfrak{f}=0$, the expected dimension of $\mathfrak{O}_{0} \subset \overline{\mathfrak{M}}_{0,0}(\overline{\mathfrak{M}}(n), d \mathfrak{f})$ is $4 n-7$ by (2.2). Hence the excess dimension of $\mathfrak{O}_{0}$ is $e=2 d-1$.

Let $\mathcal{V}$ be the restriction of $R^{1}\left(f_{1,0}\right)_{*}\left(e v_{1}\right)^{*} T_{\overline{\mathfrak{M}}(n)}$ to $\mathfrak{O}_{0}$.

Lemma 5.1. (i) The sheaf $\mathcal{V}$ is locally free of rank $2 d-1$;

(ii) $\mathcal{V} \cong R^{1}\left(\tilde{f}_{1,0}\right)_{*}\left(\widetilde{e v}_{1}\right)^{*} \mathcal{O}_{\mathfrak{B}_{*}}\left(\mathfrak{B}_{*}\right)$.

Proof. (i) Take a stable map $u=[\mu: D \rightarrow \overline{\mathfrak{M}}(n)]$ in $\mathfrak{O}_{0}$, and consider

$$
H^{1}\left(f_{1,0}^{-1}(u),\left.\left(e v_{1}^{*} T_{\overline{\mathfrak{M}}(n)}\right)\right|_{f_{1,0}^{-1}(u)}\right) \cong H^{1}\left(D, \mu^{*} T_{\overline{\mathfrak{M}}(n)}\right) .
$$

Since $\mu(D) \cong \mathbb{P}^{1}$ is a fiber of the projection $\pi$, we see from Lemma 3.4 (iii) that

$$
\left.T_{\overline{\mathfrak{M}}(n)}\right|_{\mu(D)}=\mathcal{O}_{\mu(D)}^{\oplus(4 n-6)} \oplus \mathcal{O}_{\mu(D)}(-2) \oplus \mathcal{O}_{\mu(D)}(2) .
$$

Thus $H^{1}\left(D, \mu^{*} T_{\overline{\mathfrak{M}}(n)}\right) \cong H^{1}\left(D, \mu^{*} \mathcal{O}_{\mu(D)}(-2)\right)$ whose dimension equals the excess dimension $e=2 d-1$. Therefore, the restriction $\mathcal{V}$ of $R^{1}\left(f_{1,0}\right)_{*}\left(e v_{1}\right)^{*} T_{\overline{\mathfrak{M}}(n)}$ to $\mathfrak{O}_{0}$ is a locally free sheaf of rank $2 d-1$.

(ii) Since $e v_{1}\left(\mathfrak{O}_{1}\right) \subset \mathfrak{B}_{*}$, we have $\left.\left(\left(e v_{1}\right)^{*} T_{\overline{\mathfrak{M}}(n)}\right)\right|_{\mathfrak{O}_{1}}=\left(\widetilde{e v_{1}}\right)^{*}\left(\left.T_{\overline{\mathfrak{M}}_{*}(n)}\right|_{\mathfrak{B}_{*}}\right)$ and

$$
\begin{aligned}
\mathcal{V} & =\left.\left(R^{1}\left(f_{1,0}\right)_{*}\left(e v_{1}\right)^{*} T_{\overline{\mathfrak{M}}(n)}\right)\right|_{\mathfrak{O}_{0}}=R^{1}\left(\tilde{f}_{1,0}\right)_{*}\left(\left.\left(\left(e v_{1}\right)^{*} T_{\overline{\mathfrak{M}}(n)}\right)\right|_{\mathfrak{O}_{1}}\right) \\
& =R^{1}\left(\tilde{f}_{1,0}\right)_{*}\left(\widetilde{e v}_{1}\right)^{*}\left(\left.T_{\overline{\mathfrak{M}}(n)}\right|_{\mathfrak{B}_{*}}\right) .
\end{aligned}
$$

Since $\mathfrak{B}_{*}$ is smooth of codimension 1 in $\overline{\mathfrak{M}}(n)$, we obtain the exact sequence

$$
\left.0 \rightarrow T_{\mathfrak{B}_{*}} \rightarrow T_{\overline{\mathfrak{M}}(n)}\right|_{\mathfrak{B}_{*}} \rightarrow \mathcal{O}_{\mathfrak{B}_{*}}\left(\mathfrak{B}_{*}\right) \rightarrow 0
$$

Applying $\left(\widetilde{e v}_{1}\right)^{*}$ and $\left(\tilde{f}_{1,0}\right)_{*}$ to the exact sequence (5.2), we get

$$
R^{1}\left(\tilde{f}_{1,0}\right)_{*}\left(\widetilde{e v}_{1}\right)^{*} T_{\mathfrak{B}_{*}} \rightarrow \mathcal{V} \rightarrow R^{1}\left(\tilde{f}_{1,0}\right)_{*}\left(\widetilde{e v}_{1}\right)^{*} \mathcal{O}_{\mathfrak{B}_{*}}\left(\mathfrak{B}_{*}\right) \rightarrow 0
$$

where we have used $R^{2}\left(\tilde{f}_{1,0}\right)_{*}\left(\widetilde{e v}_{1}\right)^{*} T_{\mathfrak{B}_{*}}=0$ since $\tilde{f}_{1,0}$ is of relative dimension 1 . 
If $[\mu: D \rightarrow \overline{\mathfrak{M}}(n)]$ is a stable map in $\mathfrak{O}_{0}$, then $\mu(D)$ is a fiber of the projection $\pi$ in (3.9). Hence the normal bundle of $\mu(D)$ in $\mathfrak{B}_{*}$ is trivial. Therefore we have

$$
\left.T_{\mathfrak{B}_{*}}\right|_{\mu(D)} \cong \mathcal{O}_{\mu(D)}^{\oplus(4 n-6)} \oplus \mathcal{O}_{\mu(D)}(2)
$$

and

$$
H^{1}\left(D, \mu^{*} T_{\mathfrak{B}_{*}}\right) \cong H^{1}\left(D, \mu^{*}\left(\mathcal{O}_{\mu(D)}^{\oplus(4 n-6)} \oplus \mathcal{O}_{\mu(D)}(2)\right)\right)=0 .
$$

It follows that $R^{1}\left(\tilde{f}_{1,0}\right)_{*}\left(\widetilde{e v}_{1}\right)^{*} T_{\mathfrak{B}_{*}}=0$.

Proposition 5.2. Put $\mathcal{L}=\operatorname{det}\left(\mathcal{E}_{n-1}^{0}\right)$. Then $\mathcal{V}$ sits in the exact sequence

$$
\begin{gathered}
0 \rightarrow \phi^{*}\left(\mathcal{L}^{-1} \otimes \tilde{\pi}_{1}^{*} \mathcal{O}_{\mathfrak{M}(n-1)}\left(\mathfrak{D}_{n-1}\right) \otimes \tilde{\pi}_{2}^{*} \mathcal{O}_{X}(2 \ell)\right) \rightarrow \mathcal{V} \\
\rightarrow R^{1}\left(\tilde{f}_{1,0}\right)_{*} \widetilde{e v}_{1}^{*}\left(\pi^{*}\left(\mathcal{E}_{n-1}^{0}\right)^{*} \otimes \mathcal{O}_{\mathfrak{B}_{*}}(-1)\right) \otimes \phi^{*}\left(\tilde{\pi}_{1}^{*} \mathcal{O}_{\mathfrak{M}(n-1)}\left(\mathfrak{D}_{n-1}\right) \otimes \tilde{\pi}_{2}^{*} \mathcal{O}_{X}(2 \ell)\right) \rightarrow 0 .
\end{gathered}
$$

Proof. Recall that $\mathfrak{B}_{*}=\mathbb{P}\left(\mathcal{E}_{n-1}^{0}\right)$. The kernel of the tautological surjection $\pi^{*} \mathcal{E}_{n-1}^{0}$ $\rightarrow \mathcal{O}_{\mathfrak{B}_{*}}(1) \rightarrow 0$ is a line bundle. By comparing the first Chern classes, we get

$$
0 \rightarrow \pi^{*} \mathcal{L} \otimes \mathcal{O}_{\mathfrak{B}_{*}}(-1) \rightarrow \pi^{*} \mathcal{E}_{n-1}^{0} \rightarrow \mathcal{O}_{\mathfrak{B}_{*}}(1) \rightarrow 0 .
$$

Tensoring with $\pi^{*} \mathcal{L}^{-1} \otimes \mathcal{O}_{\mathfrak{B}_{*}}(-1)$, we obtain the exact sequence

$$
0 \rightarrow \mathcal{O}_{\mathfrak{B}_{*}}(-2) \rightarrow \pi^{*}\left(\mathcal{E}_{n-1}^{0} \otimes \mathcal{L}^{-1}\right) \otimes \mathcal{O}_{\mathfrak{B}_{*}}(-1) \rightarrow \pi^{*} \mathcal{L}^{-1} \rightarrow 0 .
$$

Note that $\mathcal{E}_{n-1}^{0} \otimes \mathcal{L}^{-1} \cong\left(\mathcal{E}_{n-1}^{0}\right)^{*}$. Applying $\widetilde{e v}_{1}^{*}$ to the above exact sequence yields

$$
0 \rightarrow \widetilde{e v_{1}^{*}} \mathcal{O}_{\mathfrak{B}_{*}}(-2) \rightarrow \widetilde{e v}_{1}^{*}\left(\pi^{*}\left(\mathcal{E}_{n-1}^{0}\right)^{*} \otimes \mathcal{O}_{\mathfrak{B}_{*}}(-1)\right) \rightarrow\left(\pi \circ \widetilde{e v}_{1}\right)^{*} \mathcal{L}^{-1} \rightarrow 0 .
$$

By (5.1), $\pi \circ \widetilde{e v}_{1}=\phi \circ \tilde{f}_{1,0}$. Rewriting the 3rd term, we have

Applying the functor $\left(\tilde{f}_{1,0}\right)_{*}$ to (5.4), we get the exact sequence

$$
\begin{aligned}
0 & \rightarrow \phi^{*} \mathcal{L}^{-1} \rightarrow R^{1}\left(\tilde{f}_{1,0}\right)_{*}\left(\widetilde{e v}_{1}\right)^{*} \mathcal{O}_{\mathfrak{B}_{*}}(-2) \\
& \rightarrow R^{1}\left(\tilde{f}_{1,0}\right)_{*} \widetilde{e v_{1}^{*}}\left(\pi^{*}\left(\mathcal{E}_{n-1}^{0}\right)^{*} \otimes \mathcal{O}_{\mathfrak{B}_{*}}(-1)\right) \rightarrow 0
\end{aligned}
$$

where we have used the projection formula, $\left(\tilde{f}_{1,0}\right)_{*} \mathcal{O}_{\mathfrak{O}_{1}} \cong \mathcal{O}_{\mathfrak{O}_{0}}$, and

$$
R^{1}\left(\tilde{f}_{1,0}\right)_{*} \mathcal{O}_{\mathfrak{O}_{1}}=0, \quad\left(\tilde{f}_{1,0}\right)_{*} \widetilde{e v}_{1}^{*}\left(\pi^{*}\left(\mathcal{E}_{n-1}^{0}\right)^{*} \otimes \mathcal{O}_{\mathfrak{B}_{*}}(-1)\right)=0 .
$$

By Lemma 5.1 and Lemma 3.3, we obtain the desired exact sequence for $\mathcal{V}$.

Remark 5.3. Fix a point $\left(V_{1}, x\right) \in \mathfrak{M}(n-1) \times X$. Via $\phi^{-1}\left(V_{1}, x\right) \cong \overline{\mathfrak{M}}_{0,0}\left(\mathbb{P}^{1}, d\left[\mathbb{P}^{1}\right]\right)$, the restriction of $R^{1}\left(\tilde{f}_{1,0}\right)_{*} \widetilde{e v}_{1}^{*}\left(\pi^{*}\left(\mathcal{E}_{1}^{0}\right)^{*} \otimes \mathcal{O}_{\mathfrak{B}_{*}}(-1)\right)$ to $\phi^{-1}\left(V_{1}, x\right)$ is isomorphic to

$$
R^{1}\left(f_{1,0}\right)_{*}\left(e v_{1}\right)^{*}\left(\mathcal{O}_{\mathbb{P}^{1}}(-1) \oplus \mathcal{O}_{\mathbb{P}^{1}}(-1)\right),
$$

where by abusing notation, we still use $f_{1,0}$ and $e v_{1}$ to denote the forgetful map and the evaluation map from $\overline{\mathfrak{M}}_{0,1}\left(\mathbb{P}^{1}, d\left[\mathbb{P}^{1}\right]\right)$ to $\overline{\mathfrak{M}}_{0,0}\left(\mathbb{P}^{1}, d\left[\mathbb{P}^{1}\right]\right)$ and $\mathbb{P}^{1}$, respectively. 


\section{The virtual Fundamental CLAss $\left[\overline{\mathfrak{M}}_{0,1}(\overline{\mathfrak{M}}(n), d \mathfrak{f})\right]^{\text {vir }}$}

As we saw in the construction of the classes $\Xi_{i}, \Xi_{5}$ and $\Xi_{6}$ don't lie in $\mathfrak{B}_{*}$. The method to compute the virtual cycle restricted to $e v_{1}^{-1}\left(\Xi_{i}\right)$ in the previous section won't work for $i=5,6$. In this section, we shall employ the localization method of Kiem-Li to find a sufficiently small closed subset of $\overline{\mathfrak{M}}(n)$ containing the image of the virtual cycle under the evaluation map $e v_{1}$. The result will be used to show the vanishing of the Gromov-Witten invariants $\langle\alpha\rangle_{0, d \mathfrak{f}}$ when $\alpha$ is dual to $\Xi_{5}, \Xi_{6}$.

Let $X=\mathbb{P}^{2}$, and let $C_{0} \subset X$ be a smooth cubic curve. Recall that the Zariski tangent space of $\overline{\mathfrak{M}}(n)$ at $V \in \overline{\mathfrak{M}}(n)$ is canonically $\operatorname{Ext}^{1}(V, V)$. Therefore, the natural map $K_{X}=\mathcal{O}_{X}\left(-C_{0}\right) \rightarrow \mathcal{O}_{X}$ induces a meromorphic 2-form $\Theta$ on the moduli space $\overline{\mathfrak{M}}(n)$ given point-wisely by

$$
\operatorname{Ext}^{1}(V, V)^{*} \cong \operatorname{Ext}^{1}\left(V, V \otimes K_{X}\right) \rightarrow \operatorname{Ext}^{1}(V, V) .
$$

Note that $\Theta$ is holomorphic at $V \in \overline{\mathfrak{M}}(n)$ if (6.1) is an isomorphism.

The constructions in $\mathrm{K}-\mathrm{L}, \mathrm{L}-\mathrm{L}$, show that the meromorphic 2-form $\Theta$ on the moduli space $\overline{\mathfrak{M}}(n)$ induces a meromorphic homomorphism:

$$
\eta: \mathcal{E} \rightarrow \mathcal{O}
$$

over $\overline{\mathfrak{M}}_{0,1}(\overline{\mathfrak{M}}(n), d \mathfrak{f})$. Here $\mathcal{E}$ is a suitable bundle on $\overline{\mathfrak{M}}_{0,1}(\overline{\mathfrak{M}}(n), d \mathfrak{f})$ such that

$$
\left[\overline{\mathfrak{M}}_{0,1}(\overline{\mathfrak{M}}(n), d \mathfrak{f})\right]^{\text {vir }} \in H_{2(4 n-6)}(\Lambda),
$$

where $\Lambda \subset \overline{\mathfrak{M}}_{0,1}(\overline{\mathfrak{M}}(n), d \mathfrak{f})$ is the degeneracy loci consisting of points at which either the map $\eta$ is undefined or not surjective.

Next, we analyze the degeneracy loci $\Lambda$. Let $[\mu:(D ; p) \rightarrow \overline{\mathfrak{M}}(n)] \in \Lambda$. Since $\mu_{*}[D]=d \mathfrak{f}, \mu(D)$ is contracted to a point by the Gieseker-Uhlenbeck morphism $\Psi$.

Lemma 6.1. Let $[\mu:(D ; p) \rightarrow \overline{\mathfrak{M}}(n)] \in \Lambda$, and put

$$
(\Psi \circ \mu)(D)=\left(V_{k} ; \zeta\right) \in \mathfrak{M}(n-k) \times \operatorname{Sym}^{k}(X) \subset \overline{\mathfrak{U}}(n)
$$

for some $1 \leq k \leq(n-1)$. Then, either $\operatorname{Supp}(\zeta) \cap C_{0} \neq \emptyset$ or $\left.V_{k}\right|_{C_{0}}$ is not stable.

Proof. Assume that $\operatorname{Supp}(\zeta) \cap C_{0}=\emptyset$ and $\left.V_{k}\right|_{C_{0}}$ is stable. We will draw a contradiction by showing that $\eta$ is both defined and surjective at $[\mu:(D ; p) \rightarrow \overline{\mathfrak{M}}(n)]$.

Note that $\eta$ is defined at $[\mu:(D ; p) \rightarrow \overline{\mathfrak{M}}(n)]$ if $\Theta$ is holomorphic along $\mu(D)$. Let $V \in \mu(D)$. Then, $V^{* *}=V_{k}$ and we have $0 \rightarrow V \rightarrow V_{k} \rightarrow Q \rightarrow 0$, where $Q$ is a torsion sheaf with $\sum_{x \in X} h^{0}\left(X, Q_{x}\right) x=\zeta$. So $\left.\left.V\right|_{C_{0}} \cong V_{k}\right|_{C_{0}}$ is locally free and stable. From $0 \rightarrow K_{X} \rightarrow \mathcal{O}_{X} \rightarrow \mathcal{O}_{C_{0}} \rightarrow 0$, we get $\left.0 \rightarrow V \otimes K_{X} \rightarrow V \rightarrow V\right|_{C_{0}} \rightarrow 0$. Applying the functor $\operatorname{Hom}(V, \cdot)$, we obtain a long exact sequence:

$$
\begin{gathered}
0 \rightarrow \operatorname{Hom}\left(V, V \otimes K_{X}\right) \rightarrow \operatorname{Hom}(V, V) \rightarrow \operatorname{Hom}\left(V,\left.V\right|_{C_{0}}\right) \\
\rightarrow \operatorname{Ext}^{1}\left(V, V \otimes K_{X}\right) \rightarrow \operatorname{Ext}^{1}(V, V) .
\end{gathered}
$$

Since $V$ and $\left.V_{k}\right|_{C_{0}}$ are stable, we have $\operatorname{Hom}(V, V) \cong \mathbb{C}, \operatorname{Hom}\left(V, V \otimes K_{X}\right)=0$ and $\operatorname{Hom}\left(V,\left.V\right|_{C_{0}}\right) \cong \operatorname{Hom}\left(\left.V_{k}\right|_{C_{0}},\left.V_{k}\right|_{C_{0}}\right) \cong \mathbb{C}$. The above exact sequence is simplified to

$$
0 \rightarrow \operatorname{Ext}^{1}\left(V, V \otimes K_{X}\right) \rightarrow \operatorname{Ext}^{1}(V, V) .
$$

Since $\operatorname{dim} \operatorname{Ext}^{1}\left(V, V \otimes K_{X}\right)=\operatorname{dim}_{\operatorname{Ext}^{1}}(V, V)$, we obtain an isomorphism

$$
\operatorname{Ext}^{1}\left(V, V \otimes K_{X}\right) \cong \operatorname{Ext}^{1}(V, V) \text {. }
$$

Hence the meromorphic 2-form $\Theta$ is defined at $V \in \mu(D)$. This proves that $\Theta$ is holomorphic along $\mu(D)$. So $\eta$ is defined at $[\mu:(D ; p) \rightarrow \overline{\mathfrak{M}}(n)]$. 
The above argument also shows that $\left.\Theta\right|_{\mu(D)}$ is an isomorphism. Since $\mu$ is not a constant map, the image of $\mu_{*}: T_{D_{\text {reg }}} \rightarrow T_{\overline{\mathfrak{M}}(n)}$ does not lie in the null space of $\Theta: T_{\overline{\mathfrak{M}}(n)} \rightarrow\left(T_{\overline{\mathfrak{M}}(n)}\right)^{*}$, where $D_{\text {reg }}$ denotes the smooth part of $D$. By the vanishing criterion in $[\underline{\mathrm{K}-\mathrm{L}}, \eta$ is surjective at $[\mu:(D ; p) \rightarrow \overline{\mathfrak{M}}(n)]$.

Lemma 6.2. Let $C_{0} \subset X=\mathbb{P}^{2}$ be a smooth cubic curve. Let $n \geq 1$, and let $V \in \overline{\mathfrak{M}}(n)$ be generic. Then, the restriction $\left.V\right|_{C_{0}}$ is stable.

Proof. It is well known that the cotangent bundle $\Omega_{X}$ is stable. So $\Omega_{X} \otimes \mathcal{O}_{X}(1) \in$ $\overline{\mathfrak{M}}(1)$. Let $\xi$ consist of $n-1$ distinct points away from $C_{0}$. Choose a surjection $\Omega_{X} \otimes \mathcal{O}_{X}(1) \rightarrow \mathcal{O}_{\xi}$, and let $V_{0}$ be the kernel. Then $V_{0} \in \overline{\mathfrak{M}}(n)$ and $\left.V_{0}\right|_{C_{0}} \cong$ $\left.\left(\Omega_{X} \otimes \mathcal{O}_{X}(1)\right)\right|_{C_{0}}$. Since $\overline{\mathfrak{M}}(n)$ is irreducible and the open subset $\mathfrak{M}(n)$ is nonempty, our lemma will follow if we can prove that $\left.\left(\Omega_{X} \otimes \mathcal{O}_{X}(1)\right)\right|_{C_{0}}$ is stable.

Let $\mathcal{O}_{C_{0}}(D)$ be any sub-line-bundle of $\left.\left(\Omega_{X} \otimes \mathcal{O}_{X}(1)\right)\right|_{C_{0}}$. Note that the degree of $\left.\left(\Omega_{X} \otimes \mathcal{O}_{X}(1)\right)\right|_{C_{0}}$ is -3 , and there is an exact sequence

$$
\left.\left.0 \rightarrow\left(\Omega_{X} \otimes \mathcal{O}_{X}(1)\right)\right|_{C_{0}} \rightarrow \mathcal{O}_{C_{0}}^{\oplus 3} \rightarrow \mathcal{O}_{X}(1)\right|_{C_{0}} \rightarrow 0
$$

induced from the exact sequence $0 \rightarrow \Omega_{X} \rightarrow \mathcal{O}_{X}(-1)^{\oplus 3} \rightarrow \mathcal{O}_{X} \rightarrow 0$. Thus

$$
\operatorname{deg}(D) \leq 0 \text {. }
$$

If $\operatorname{deg}(D)=0$, then $D$ must be the trivial divisor and $\mathcal{O}_{C_{0}}(D)=\mathcal{O}_{C_{0}}$. So $H^{0}\left(C_{0},\left.\left(\Omega_{X} \otimes \mathcal{O}_{X}(1)\right)\right|_{C_{0}}\right) \neq 0$. On the other hand, (6.3) induces an exact sequence

$$
0 \rightarrow H^{0}\left(C_{0},\left.\left(\Omega_{X} \otimes \mathcal{O}_{X}(1)\right)\right|_{C_{0}}\right) \rightarrow H^{0}\left(C_{0}, \mathcal{O}_{C_{0}}^{\oplus 3}\right) \stackrel{f_{0}}{\rightarrow} H^{0}\left(C_{0},\left.\mathcal{O}_{X}(1)\right|_{C_{0}}\right) .
$$

The image of $f_{0}$ is $\left.H^{0}\left(X, \mathcal{O}_{X}(1)\right)\right|_{C_{0}}=H^{0}\left(C_{0},\left.\mathcal{O}_{X}(1)\right|_{C_{0}}\right)$. So $f_{0}$ is surjective. Since $H^{0}\left(C_{0}, \mathcal{O}_{C_{0}}^{\oplus 3}\right)$ and $H^{0}\left(C_{0},\left.\mathcal{O}_{X}(1)\right|_{C_{0}}\right)$ have the same dimension, $f_{0}$ is an isomorphism and $H^{0}\left(C_{0},\left.\left(\Omega_{X} \otimes \mathcal{O}_{X}(1)\right)\right|_{C_{0}}\right)=0$. Hence we obtain a contradiction.

If $\operatorname{deg}(D)=-1$, then $\mathcal{O}_{C_{0}}(D)=\mathcal{O}_{C_{0}}(-x)$ for a unique $x \in C_{0}$. So the map

$$
H^{0}\left(C_{0}, \mathcal{O}_{C_{0}}(x)^{\oplus 3}\right) \stackrel{f_{7}}{\rightarrow} H^{0}\left(C_{0},\left.\mathcal{O}_{X}(1)\right|_{C_{0}} \otimes \mathcal{O}_{C_{0}}(x)\right)
$$

induced from (6.3) is not injective. On the other hand, since $H^{0}\left(C_{0}, \mathcal{O}_{C_{0}}(x)\right) \cong \mathbb{C}$,

$$
\begin{aligned}
\operatorname{im}\left(f_{1}\right) & =\left.H^{0}\left(X, \mathcal{O}_{X}(1)\right)\right|_{C_{0}} \otimes H^{0}\left(C_{0}, \mathcal{O}_{C_{0}}(x)\right) \\
& =H^{0}\left(C_{0},\left.\mathcal{O}_{X}(1)\right|_{C_{0}}\right) \otimes H^{0}\left(C_{0}, \mathcal{O}_{C_{0}}(x)\right) \\
& \subset H^{0}\left(C_{0},\left.\mathcal{O}_{X}(1)\right|_{C_{0}} \otimes \mathcal{O}_{C_{0}}(x)\right) .
\end{aligned}
$$

So the linear system corresponding to $\operatorname{im}\left(f_{1}\right)$ consists of all the elements $\left.\ell\right|_{C_{0}}+x$, where $\ell$ denotes lines in $X$. In particular, the dimension of $\operatorname{im}\left(f_{1}\right)$ is 3 . Thus $f_{1}$ must be injective. Again, we obtain a contradiction.

By (6.4), $\operatorname{deg}(D) \leq-2$. Therefore, $\left.\left(\Omega_{X} \otimes \mathcal{O}_{X}(1)\right)\right|_{C_{0}}$ is stable.

Definition 6.3. For $n \geq 2$, we define $\mathfrak{T}_{C_{0}}(n)$ (respectively, $\mathfrak{U}_{C_{0}}(n)$ ) to be the subset of $\overline{\mathfrak{M}}(n)$ consisting of all the nonlocally free sheaves $V$ such that $\left.V\right|_{C_{0}}$ contains torsion (respectively, $\left.V\right|_{C_{0}}$ is torsion-free and unstable).

Lemma 6.4. Let $V \in \overline{\mathfrak{M}}(n)-\mathfrak{M}(n)$ and $\Psi(V)=\left(V_{k} ; \zeta\right)$. Then,

(i) $V \in \mathfrak{T}_{C_{0}}(n)$ if and only if $\operatorname{Supp}(\zeta) \cap C_{0} \neq \emptyset$;

(ii) $V \in \mathfrak{U}_{C_{0}}(n)$ if and only if $\operatorname{Supp}(\zeta) \cap C_{0}=\emptyset$ and $\left.V_{k}\right|_{C_{0}}$ is unstable.

Lemma 6.5. (i) $\left(e v_{1}\right)(\Lambda) \subset \mathfrak{T}_{C_{0}}(n) \amalg \mathfrak{U}_{C_{0}}(n)$.

(ii) Both $\mathfrak{T}_{C_{0}}(n)$ and $\mathfrak{T}_{C_{0}}(n) \amalg \mathfrak{U}_{C_{0}}(n)$ are closed subsets of $\overline{\mathfrak{M}}(n)$. 
Proof. By Lemma 6.1, the image of a point in $\left(e v_{1}\right)(\Lambda)$ under $\Psi$ is of the form

$$
\left(V_{k} ; \zeta\right) \in \mathfrak{M}(n-k) \times \operatorname{Sym}^{k}(X) \subset \overline{\mathfrak{U}}(n),
$$

where $1 \leq k \leq n-1$, and either $\operatorname{Supp}(\zeta) \cap C_{0} \neq \emptyset$ or $\left.V_{k}\right|_{C_{0}}$ is not stable. So we see from Lemma 6.4 that $\left(e v_{1}\right)(\Lambda) \subset \mathfrak{T}_{C_{0}}(n) \amalg \mathfrak{U}_{C_{0}}(n)$. This proves (i).

Since being torsion-free and being stable are open conditions, both $\mathfrak{T}_{C_{0}}(n)$ and $\mathfrak{T}_{C_{0}}(n) \amalg \mathfrak{U}_{C_{0}}(n)$ are closed subsets of $\overline{\mathfrak{M}}(n)$. This proves (ii).

\section{The 1-Point Gromov-Witten InVARIANTS}

Now we are ready to compute the 1-point Gromov-Witten invariants

$$
\langle\alpha\rangle_{0, d \mathfrak{f}}=\int_{\left[\overline{\mathfrak{M}}_{0,1}(\overline{\mathfrak{M}}(n), d \mathfrak{f})\right]^{\text {vir }}} e v_{1}^{*} \alpha,
$$

where $\alpha \in H^{8 n-12}(\overline{\mathfrak{M}}(n) ; \mathbb{C})$ denotes the Poincaré duals of the classes $\Xi_{1}, \ldots, \Xi_{6} \in$ $H_{4}(\overline{\mathfrak{M}}(n) ; \mathbb{C})$. By abusing notation, we use $\Xi_{i}$ to stand for both the class in $H_{4}(\overline{\mathfrak{M}}(n) ; \mathbb{C})$ and its Poincaré dual in $H^{8 n-12}(\overline{\mathfrak{M}}(n) ; \mathbb{C})$.

Lemma 7.1. $\left\langle\Xi_{1}\right\rangle_{0, d \mathfrak{f}}=-6 / d^{2}$.

Proof. Let $\alpha=\Xi_{1}$. By Subsection 4.1] $\Xi_{1}=\pi^{-1}\left(\left\{V_{1}\right\} \times \ell\right)=\mathbb{P}\left(\left.V_{1}\right|_{\ell}\right)$, where the stable vector bundle $V_{1} \in \mathfrak{M}(n-1)$ and the line $\ell \subset X$ are fixed. Let $\mathfrak{M}=$ $\left(e v_{1}\right)^{-1}\left(\Xi_{1}\right)$. Then $\mathfrak{M} \subset \mathfrak{O}_{1}$. By Proposition 5.2 and Proposition 2.1 for $k=1$ and $\mathfrak{O}=\mathfrak{O}_{1}$, we obtain

$$
\begin{aligned}
\langle\alpha\rangle_{0, d \mathfrak{f}} & \left.=\int_{\left[\overline{\mathfrak{M}}_{0,1}(\overline{\mathfrak{M}}(n), d \mathfrak{f})\right.}\right]^{\mathrm{vir}}\left(e v_{1}\right)^{*} \alpha=\int_{\left(\tilde{f}_{1,0}\right)^{*} c_{2 d-1}(\mathcal{V})}\left(e v_{1}\right)^{*} \alpha \\
& =\int_{\left(\tilde{f}_{1,0}\right)^{*} \phi^{*}\left(-\mathcal{L}+\tilde{\pi}_{1}^{*} \mathfrak{D}_{n-1}+2 \tilde{\pi}_{2}^{*} \ell\right) \cdot\left(\tilde{f}_{1,0}\right)^{*} c_{2 d-2}(\mathbb{E})}\left(e v_{1}\right)^{*} \alpha,
\end{aligned}
$$

where $\mathbb{E}=R^{1}\left(\tilde{f}_{1,0}\right)_{*} \widetilde{e v}_{1}^{*}\left(\pi^{*}\left(\mathcal{E}_{1}^{0}\right)^{*} \otimes \mathcal{O}_{\mathfrak{B}_{*}}(-1)\right) \otimes \phi^{*}\left(\tilde{\pi}_{1}^{*} \mathcal{O}_{\mathfrak{M}(n-1)}\left(\mathfrak{D}_{n-1}\right) \otimes \tilde{\pi}_{2}^{*} \mathcal{O}_{X}(2 \ell)\right)$. Note that $\Xi_{1} \subset \mathfrak{B}_{*}$ and $\phi \circ \tilde{f}_{1,0}=\pi \circ \widetilde{e v}_{1}$. So we obtain

$$
\begin{aligned}
& \langle\alpha\rangle_{0, d \mathfrak{f}} \\
= & \left(\widetilde{e v}_{1}\right)^{*} \pi^{*}\left(-\mathcal{L}+\tilde{\pi}_{1}^{*} \mathfrak{D}_{n-1}+2 \tilde{\pi}_{2}^{*} \ell\right) \cdot\left(\tilde{f}_{1,0}\right)^{*} c_{2 d-2}(\mathbb{E}) \cdot\left(\widetilde{e v}_{1}\right)^{*}\left(\left[\Xi_{1}\right] \cdot c_{1}\left(\mathcal{O}_{\mathfrak{B}_{*}}\left(\mathfrak{B}_{*}\right)\right)\right) \\
= & \left(\tilde{f}_{1,0}\right)^{*} c_{2 d-2}(\mathbb{E}) \cdot\left(\widetilde{e v}_{1}\right)^{*}\left(\pi^{*}\left(-\mathcal{L}+\tilde{\pi}_{1}^{*} \mathfrak{D}_{n-1}+2 \tilde{\pi}_{2}^{*} \ell\right) \cdot\left[\Xi_{1}\right] \cdot c_{1}\left(\mathcal{O}_{\mathfrak{B}_{*}}\left(\mathfrak{B}_{*}\right)\right)\right) .
\end{aligned}
$$

Recall the definitions of $\mathcal{L}$ and $\mathfrak{D}_{n-1}$ in Proposition 5.2 and Lemma 3.3. We have

$$
\begin{gathered}
\mathcal{L}=\operatorname{det}\left(\widetilde{\mathcal{E}}_{n-1}\right)=(2,-1) \in \operatorname{Pic}(\Gamma \times X), \\
\mathfrak{D}_{n-1}=2 a_{n-1,1}-a_{n-1,2}-a_{n-1,0}=2 \in \operatorname{Pic}(\Gamma), \\
\left.c_{1}\left(\mathcal{O}_{\mathfrak{B}_{*}}\left(\mathfrak{B}_{*}\right)\right)\right|_{\mathbb{P}}=-2 c_{1}\left(\mathcal{O}_{\mathbb{P}}(1)\right)+\pi^{*}(2,2) \in \operatorname{Pic}(\mathbb{P})
\end{gathered}
$$

in view of the exact sequence (4.1), the degrees in (4.3) and Lemma 3.3. Thus,

$$
\langle\alpha\rangle_{0, d \mathfrak{f}}=\left(\tilde{f}_{1,0}\right)^{*} c_{2 d-2}(\mathbb{E}) \cdot\left(\widetilde{e v}_{1}\right)^{*}\left(\pi^{*}(0,3) \cdot\left[\Xi_{1}\right] \cdot\left(-2 c_{1}\left(\mathcal{O}_{\mathbb{P}}(1)\right)+\pi^{*}(2,2)\right)\right) .
$$

Since $\left[\Xi_{1}\right]=\pi^{*}\left(\left\{V_{1}\right\} \times \ell\right)$, it follows immediately that

$$
\langle\alpha\rangle_{0, d \mathfrak{f}}=-6 \cdot\left(\tilde{f}_{1,0}\right)^{*} c_{2 d-2}(\mathbb{E}) \cdot\left(\widetilde{e v}_{1}\right)^{*}[\xi],
$$

where $[\xi]$ denotes the cycle of a fixed point $\xi$ in $\mathbb{P} \subset \mathfrak{B}_{*}$.

Let $\mathfrak{M}_{1}^{\prime}=\left(\widetilde{e v}_{1}\right)^{-1}(\xi)=\left(e v_{1}\right)^{-1}(\xi)$. If $[\mu:(D ; p) \rightarrow \overline{\mathfrak{M}}(n)] \in \mathfrak{M}_{1}^{\prime}$, then $\mu(p)=\xi$ and $\pi(\mu(D))=\pi(\mu(p))=\pi(\xi)=\left(V_{1}, x\right) \in \mathfrak{M}(n-1) \times X$. So $\mu(D)=\mathfrak{f}$, which 
denotes the unique fiber of $\pi$ from (3.9) containing the point $\xi \in \mathfrak{B}_{*}$. Thus the restriction of the forgetful map $\tilde{f}_{1,0}$ to $\mathfrak{M}_{1}^{\prime}$ gives a degree- $d$ morphism from $\mathfrak{M}_{1}^{\prime}$ to

$$
\mathfrak{M}_{0}^{\prime} \stackrel{\text { def }}{=} \tilde{f}_{1,0}\left(\mathfrak{M}_{1}^{\prime}\right)=\phi^{-1}\left(V_{1}, x\right)
$$

Hence, as algebraic cycles, we have $\left(\tilde{f}_{1,0}\right)_{*}\left[\mathfrak{M}_{1}^{\prime}\right]=d\left[\mathfrak{M}_{0}^{\prime}\right]=d \cdot \phi^{*}\left[\left(V_{1}, x\right)\right]$. By (7.7),

$$
\begin{aligned}
\langle\alpha\rangle_{0, d \mathfrak{f}} & =-6 \cdot c_{2 d-2}(\mathbb{E}) \cdot\left(\tilde{f}_{1,0}\right)_{*}\left[\mathfrak{M}_{1}^{\prime}\right]=-6 d \cdot c_{2 d-2}(\mathbb{E}) \cdot \phi^{*}\left[\left(V_{1}, x\right)\right] \\
& =-6 d \cdot c_{2 d-2}\left(\left.\mathbb{E}\right|_{\phi^{-1}\left(V_{1}, x\right)}\right) .
\end{aligned}
$$

By Remark $5.3,\left.\mathbb{E}\right|_{\phi^{-1}\left(V_{1}, x\right)} \cong R^{1}\left(f_{1,0}\right)_{*}\left(e v_{1}\right)^{*}\left(\mathcal{O}_{\mathbb{P}^{1}}(-1) \oplus \mathcal{O}_{\mathbb{P}^{1}}(-1)\right)$, where $f_{1,0}$ and $e v_{1}$ denote the forgetful map and the evaluation map from the moduli space $\overline{\mathfrak{M}}_{0,1}\left(\mathbb{P}^{1}, d\left[\mathbb{P}^{1}\right]\right)$ to $\overline{\mathfrak{M}}_{0,0}\left(\mathbb{P}^{1}, d\left[\mathbb{P}^{1}\right]\right)$ and $\mathbb{P}^{1}$, respectively. We have

$$
c_{2 d-2}\left(R^{1}\left(f_{1,0}\right)_{*}\left(e v_{1}\right)^{*}\left(\mathcal{O}_{\mathbb{P}^{1}}(-1) \oplus \mathcal{O}_{\mathbb{P}^{1}}(-1)\right)\right)=\frac{1}{d^{3}}
$$

by Theorem 9.2.3 in [C-K]. Therefore, $\langle\alpha\rangle_{0, d f}=-6 / d^{2}$ by (7.8).

For $i=2,3$ or 4 , we may assume that the classes $\Xi_{2}, \Xi_{3}$ and $\Xi_{4}$ are represented by complex surfaces in $\mathbb{P} \subset \mathfrak{B}_{*}$. The same proofs of (7.6) and (17.7) show that

$$
\left\langle\Xi_{i}\right\rangle_{0, d \mathfrak{f}}=a_{i} \cdot\left(\tilde{f}_{1,0}\right)^{*} c_{2 d-2}(\mathbb{E}) \cdot\left(\widetilde{e v}_{1}\right)^{*}[\xi]
$$

where $[\xi]$ denotes the cycle of a fixed point $\xi$ in $\mathbb{P} \subset \mathfrak{B}_{*}$, and

$$
a_{i}=\pi^{*}(0,3) \cdot\left[\Xi_{i}\right] \cdot\left(-2 c_{1}\left(\mathcal{O}_{\mathbb{P}}(1)\right)+\pi^{*}(2,2)\right)
$$

is the intersection number in $\mathbb{P}$. Note from the last two paragraphs in the proof of Lemma 7.1 that $\left(\tilde{f}_{1,0}\right)^{*} c_{2 d-2}(\mathbb{E}) \cdot\left(\widetilde{e v}_{1}\right)^{*}[\xi]=1 / d^{2}$. Therefore,

$$
\left\langle\Xi_{i}\right\rangle_{0, d \mathfrak{f}}=\frac{a_{i}}{d^{2}} .
$$

Theorem 7.2. Let $d \geq 1$ and $n \geq 3$. The Gromov-Witten invariants $\langle\alpha\rangle_{0, d \mathfrak{f}}$ for the classes $\alpha=\operatorname{PD}\left(\Xi_{1}\right), \ldots, \operatorname{PD}\left(\Xi_{6}\right) \in H^{8 n-12}(\overline{\mathfrak{M}}(n) ; \mathbb{C})$ are respectively equal to

$$
-6 / d^{2}, \quad 12 / d^{2}, \quad 0, \quad-6 / d^{2}, \quad 0, \quad 0 .
$$

Proof. First of all, $\left\langle\Xi_{1}\right\rangle_{0, d f}=-6 / d^{2}$ is Lemma 7.1.

Next, $\left\langle\Xi_{2}\right\rangle_{0, d f}=12 / d^{2}$ follows from the computation of the number in (7.9):

$$
a_{2}=3 \pi^{*} \ell \cdot c_{1}\left(\mathcal{O}_{\mathbb{P}\left(V_{1}\right)}(1)\right) \cdot\left(-2 c_{1}\left(\mathcal{O}_{\mathbb{P}\left(V_{1}\right)}(1)\right)+2 \pi^{*} \ell\right)=12
$$

by (4.8), where $\pi: \mathbb{P}\left(V_{1}\right) \rightarrow X$ denotes the tautological projection.

Since $\Xi_{3}=\pi^{-1}(\Gamma \times\{y\})$ with $y \in X,\left\langle\Xi_{3}\right\rangle_{0, d \mathfrak{f}}=0$ comes from the computation

$$
a_{3}=\pi^{*}(0,3) \cdot\left[\Xi_{3}\right] \cdot\left(-2 c_{1}\left(\mathcal{O}_{\mathbb{P}}(1)\right)+\pi^{*}(2,2)\right)=0 .
$$

Similarly, $\left\langle\Xi_{4}\right\rangle_{0, d \mathfrak{f}}=-6 / d^{2}$ follows from the computation

$$
a_{4}=\pi^{*}(0,3) \cdot c_{1}\left(\mathcal{O}_{W}(1)\right) \cdot\left(-2 c_{1}\left(\mathcal{O}_{W}(1)\right)+\pi^{*}(2,2)\right)=-6
$$

by (4.11), where $W=\pi^{-1}(\Gamma \times \ell) \subset \mathbb{P} \subset \overline{\mathfrak{M}}(n)$ for a fixed line $\ell \subset X$.

To prove $\left\langle\Xi_{5}\right\rangle_{0, d \mathfrak{f}}=0$, choose the vector bundle $V_{2} \in \mathfrak{M}(n-2)$ and the distinct points $x_{1}, x_{2} \in X$ in Subsection 4.3 such that $\left.V_{2}\right|_{C_{0}}$ is stable and $x_{1}, x_{2} \notin C_{0}$. By Lemma 6.4, $\Xi_{5} \cap\left(\mathfrak{T}_{C_{0}}(n) \cup \mathfrak{U}_{C_{0}}(n)\right)=\emptyset$. Hence $\Xi_{5} \cap\left(e v_{1}\right)(\Lambda)=\emptyset$ by Lemma6.5 (i). Since $\left[\overline{\mathfrak{M}}_{0,1}(\overline{\mathfrak{M}}(n), d \mathfrak{f})\right]^{\text {vir }} \in H_{2(4 n-6)}(\Lambda)$, we get

$$
\left\langle\Xi_{5}\right\rangle_{0, d \mathfrak{f}}=\int_{\left[\overline{\mathfrak{M}}_{0,1}(\overline{\mathfrak{M}}(n), d \mathfrak{f})\right]^{\text {vir }}} e v_{1}^{*} \Xi_{5}=e v_{1 *}\left[\overline{\mathfrak{M}}_{0,1}(\overline{\mathfrak{M}}(n), d \mathfrak{f})\right]^{\text {vir }} \cdot \Xi_{5}=0 .
$$


Finally, to prove $\left\langle\Xi_{6}\right\rangle_{0, d \mathfrak{f}}=0$, choose the vector bundle $V_{2} \in \mathfrak{M}(n-2)$ and the point $x \in X$ in Subsection 4.4 such that $\left.V_{2}\right|_{C_{0}}$ is stable and $x \notin C_{0}$. Now our result follows from the same proof in the previous paragraph.

Remark 7.3. Let $d \geq 1$ and $n \geq 3$. Using the theorem above, one can show that

$$
e v_{1 *}\left[\overline{\mathfrak{M}}_{0,1}(\overline{\mathfrak{M}}(n), d \mathfrak{f})\right]^{\mathrm{vir}}=1 / d^{2} \mathfrak{T}_{C_{0}}(n) .
$$

\section{ACKNOWLEDGMENTS}

The authors would like to thank Jun Li for helpful discussions. We also would like to thank the referee for suggestions which helped to remove the condition $n \geq 5$ in the original draft.

\section{REFERENCES}

[Bea] A. Beauville, Sur la cohomologie de certains espaces de modules de fibrés vectoriels, Geometry and Analysis (Bombay, 1992), 37-40, Tata Inst. Fund. Res., Bombay, 1995. MR.1351502 (96f:14011)

[Beh] K. Behrend, Gromov-Witten invariants in algebraic geometry, Invent. Math. 127 (1997) 601-617. MR1431140 (98i:14015)

[B-F] K. Behrend, B. Fantechi, The intrinsic normal cone, Invent. Math. 128 (1997) 45-88. MR1437495 (98e:14022)

[C-K] D. Cox, S. Katz, Mirror symmetry and algebraic geometry, Mathematical Surveys and Monographs 68, Amer. Math. Soc., Providence, RI, 1999. MR.1677117|(2000d:14048)

[E-S] G. Ellingsrud, S. Strømme, Towards the Chow ring of the Hilbert scheme of $\mathbb{P}^{2}$, J. reine angew. Math. 441 (1993), 33-44. MR.1228610 (94i:14004)

[Ful] W. Fulton, Intersection Theory, Ergebnisse der Mathematik und ihrer Grenzgebiete 3. Folge 2. Springer, Berlin Heidelberg New York Tokyo, 1994. MR732620 (85k:14004)

[F-P] W. Fulton, R. Pandharipande, Notes on stable maps and quantum cohomology. Algebraic Geometry - Santa Cruz 1995, 45-96, Proc. Sympos. Pure Math. 62, Amer. Math. Soc., Providence, RI, 1997. MR1492534 (98m:14025)

[Get] E. Getzler, Intersection theory on $M_{1,4}$ and elliptic Gromov-Witten invariants, J. Amer. Math. Soc. 10 (1997) 973-998. MR.1451505 (98f:14018)

[Gro] A. Grothendieck, Étude cohomologique des faisceaux cohérents, Éléments de géométrie algébrique. III, Publ. Inst. Hautes Études Sci. Math, No. 17 (1963). MR0163911 $(29: 1210)$

$[\mathrm{H}-\mathrm{H}] \quad$ A. Hirschowitz, K. Hulek, Complete families of stable vector bundles over $\mathbb{P}_{2}$. With an appendix by K. Hulek and S. A. Strømme. Lecture Notes in Math. 1194, Complex Analysis and Algebraic Geometry (Gottingen, 1985), 19-40, Springer, Berlin, 1986. MR855874 (87j:14019)

[K-L] Y. Kiem, J. Li, Gromov-Witten invariants of varieties with holomorphic 2-forms. Preprint.

[LJ1] J. Li, Algebraic geometric interpretation of Donaldson's polynomial invariants, J. Differ. Geom. 37 (1993), 417-466. MR1205451 (93m:14007)

[LJ2] J. Li, Kodaira dimension of moduli space of vector bundles on surfaces, Invent. Math. 115 (1994), 1-40. MR.1248077 (94i:14016)

[L-L] J. Li, W.-P. Li, Two point extremal Gromov-Witten invariants of Hilbert schemes of points on surfaces. Preprint.

[LT1] J. Li, G. Tian, Virtual moduli cycles and Gromov-Witten invariants of algebraic varieties, J. Amer. Math. Soc. 11 (1998) 19-174. MR.1467172 (99d:14011)

[LT2] J. Li, G. Tian, Virtual moduli cycles and Gromov-Witten invariants of general symplectic manifolds, Topics in symplectic 4-manifolds (Irvine, CA, 1996), First Int. Press Lect. Ser., I, Internat. Press, Cambridge, MA, 1998, 47-83. MR1635695 (2000d:53137)

[L-Q] W.-P. Li, Z. Qin, On 1-point Gromov-Witten invariants of the Hilbert schemes of points on surfaces. Proceedings of 8th Gökova Geometry-Topology Conference (2001). Turkish J. Math. 26 (2002), 53-68. MR1892799 (2003m:14006) 
[Mar] E. Markman, Integral generators for the cohomology ring of moduli spaces of sheaves over Poisson surfaces. Adv. Math. 208 (2007), 622-646. MR.2304330 (2008b:14018)

[Mor] J.W. Morgan, Comparison of the Donaldson polynomial invariants with their algebrogeometric analogues, Topology 32 (1993), 449-488. MR.1231956 (94m:57066)

[Q-Z] Z. Qin, Q. Zhang, On the crepancy of the Gieseker-Uhlenbeck morphism. Asian J. Math. 12 (2008), 213-224. MR2439261

[Str] S.A. Strømme, Ample divisors on fine moduli spaces on the projective plane, Math. Z. 187 (1984), 405-423. MR757480 (85i:14004)

[Uhl] K. Uhlenbeck, Removable singularity in Yang-Mills fields, Comm. Math. Phys. 83 (1982), 11-29. MR648355 (83e:53034)

[Yos] K. Yoshioka, The Betti numbers of the moduli space of stable sheaves of rank 2 on $\mathbb{P}^{2}$, J. reine angew. Math. 453 (1994), 193-220. MR1285785 (95f:14022)

Department of Mathematics, Hong Kong University of Science and Technology, Clear Water Bay, Kowloon, Hong Kong

E-mail address: mawpli@ust.hk

Department of Mathematics, University of Missouri, Columbia, Missouri 65211

E-mail address: qinz@missouri.edu 